CERN-PH-TH/2010-151, UAB-FT-683, ULB-TH/10-19

\title{
Gravitational backreaction effects on the holographic phase transition
}

\author{
T. Konstandin* \\ CERN Physics Department, Theory Division, CH-1211 Geneva 23, Switzerland \\ G. Nardinit \\ Service de Physique Théorique, Université Libre de Bruxelles, 1050 Brussels, Belgium \\ M. Quiros $\ddagger$ \\ ICREA, Instituciò Catalana de Recerca i Estudis Avançats, IFAE \\ Universitat Autònoma de Barcelona, 08193 Bellaterra, Barcelona, Spain
}

(Dated: October 24, 2018)

We study radion stabilization in the compact Randall-Sundrum model by introducing a bulk scalar field, as in the Goldberger and Wise mechanism, but (partially) taking into account the backreactions from the scalar field on the metric. Our generalization reconciles the radion potential found by Goldberger and Wise with the radion mass obtained with the so-called superpotential method where backreaction is fully considered. Moreover we study the holographic phase transition and its gravitational wave signals in this model. The improved control over backreactions opens up a large region in parameter space and leads, compared to former analysis, to weaker constraints on the rank $N$ of the dual gauge theory. We conclude that, in the regime where the $1 / N$ expansion is justified, the gravitational wave signal is detectable by LISA.

*Electronic address: tkonstan@cern.ch

${ }^{\dagger}$ Electronic address: germano.nardini@ulb.ac.be

‡Electronic address: quiros@ifae.es 


\section{INTRODUCTION}

Even though the Standard Model (SM) of particle physics has achieved many impressive experimental successes it fails to provide an explanation to some experimental and theoretical issues. On the experimental side, neither the observed dark matter density can be explained (the SM lacking an appropriate natural candidate for it) nor the baryon asymmetry of the Universe can be accounted of, mainly because the SM does not provide sizeable CP violation sources and a strong enough first-order electroweak phase transition. On the theoretical side there is no plausible explanation for the huge hierarchy between the electroweak scale, responsible for the mass of the weak gauge bosons, and the Planck scale, apparent in the weakness of gravitational interactions. Thus an extension of the SM seems necessary.

Guided by the naturalness criterion to solve the Higgs hierarchy problem one very attractive possibility is Randall-Sundrum (RS) models, which are based on the framework of a compact warped extra dimension [1] with two branes localized on it: an ultraviolet (UV) brane, which provides the UV cutoff of the higher dimensional theory, and an infrared (IR) one, which spontaneously breaks the conformal symmetry of the theory. In this class of models the four-dimensional part of the metric has a strong dependence on the fifth coordinate, which is unobservable macroscopically, and the hierarchy between the two energy scales is generated by localizing the relevant physical degrees of freedom responsible for electroweak breaking at (or near) the IR brane. In this way the huge hierarchy can be explained by a natural distance between the two branes of $\mathcal{O}(10)$ times the fundamental five-dimensional Planck length.

To complete the picture the distance between the branes should not be considered as a fundamental input but instead it should arise from a stabilization mechanism. In fact this is even essential to avoid a massless radion generating an unobserved fifth force, and to obtain the observed Friedman-Robertson-Waker cosmology at late times [2, 3]. One elegant possibility to stabilize the brane distance is to assume the existence of a bulk scalar with a five-dimensional mass ${ }^{1}$ that is slightly smaller than the fundamental Planck mass scale, as pioneered by Goldberger and Wise [6]. In this setup one usually assumes the weak field limit in which case the dynamics of the bulk scalar field and the metric decouple. This does not

\footnotetext{
${ }^{1}$ Alternatively, Casimir effect can also lead to a stabilizing potential [4, 5].
} 
only have the advantage of simplifying the analysis but also facilitates the interpretation of the scalar action as a radion potential.

In the modern context of the AdS/CFT correspondence [7] this kind of model can be interpreted as dual to a strongly coupled gauge theory that for instance might serve as a UV completion [8] to little Higgs models [9]. Usually the five-dimensional system is considered in the limit of a large ratio between the Planck mass and the bulk cosmological constant, which implies a large number of degrees of freedom in the dual field theory and allows to neglect stringy effects. However there is a certain tension between this assumption and a viable cosmology: even though the model has the above discussed brane setup at low temperature (called RS-GW in the following), at high temperature it is represented by an AdS-Schwarzschild (AdS-S) bulk metric according to the AdS/CFT correspondence. Even though a first-order phase transition could allow the Universe to escape from the (conformal) AdS-S to today's (quasi-conformal) RS-GW phase [10], its completion leads to a stringent constraint on the above ratio [10-14] which jeopardizes the consistency of the original assumptions.

On the other hand several appealing features characterize this necessary phase transition. One of them is its extreme supercooling, which may couple the conformal phase transition to the electroweak one making the latter strong enough to plausibly explain the baryon asymmetry of the Universe [13]. Besides a further feature is its potential testability: the phase transition could mark the gravitational wave spectrum which could allow to probe most of the interesting parameter space of the model [11].

In conclusion given the success of RS models as extensions of the SM we believe it is of importance to clarify the impact of the phase transition on the validity of the models. This paper is dedicated to this issue in the case of RS-GW models. Our aim is to alleviate the tension between the ratio of the Planck mass to the bulk cosmological constant and the phase transition completion and to show how this scenario can be tested in the gravitational wave spectrum.

In this paper we will proceed as follows. After introducing some notations and conventions in sec. [II we will review in sec. [III the usual procedure to determine the effect of the bulk scalar on the brane distance [6, 15]. We will remark that the method of ref. [6] determines the effective potential of the radion although without taking into account the backreactions of the scalar field on the gravitational metric. On the contrary the superpotential method [15] 
solves the problem exactly but it does not provide the radion effective potential which is needed to study the phase transition. For this reason in sec. IV we will present an alternative approach to determine the radion potential in the regime of detuned brane tensions and sizeable backreactions. It is based on fitting the radion potential information that we can determine in this regime: the position of the radion potential extrema and the radion mass and cosmological constant in these extrema. The obtained potential will be used in sec. V to study the phase transition and we will find relaxed bounds that may alleviate the above problematic parameter tension. The corresponding gravitational wave spectrum will be determined in sec. VI and we will conclude by commenting on the prospects of detection with forthcoming experiments as LISA. Finally we will devote sec. VII to summarize the main results of the paper and we leave in appendix $\mathrm{A}$ some technical details that we need in sec. IV to determine the radion mass in the presence of a cosmological constant.

\section{NOTATION AND CONVENTIONS}

A very interesting feature of RS models with a scalar in the bulk is that the scalar field can stabilize the brane distance [6, 15]. The corresponding five-dimensional (5D) action is given by

$$
S=\int d^{5} x \sqrt{\left|\operatorname{det} g_{M N}\right|}\left[-M^{3} R+\frac{1}{2}(\partial \phi)^{2}-V(\phi)\right]-\sum_{\alpha} \int_{B_{\alpha}} d^{4} x \sqrt{\left|\operatorname{det} \bar{g}_{\mu \nu}\right|} \lambda_{\alpha}(\phi)
$$

where $M$ is the 5D Planck scale, $\lambda_{\alpha}$ and $V$ are the brane and bulk potentials of the scalar field $\phi$, and the metric $g_{M N}$ is defined in proper coordinates by

$$
\begin{aligned}
& d s^{2}=e^{2 A(r)} \bar{g}_{\mu \nu} d x^{\mu} d x^{\nu}-d r^{2}, \\
& \mathcal{M}_{4}: \quad \bar{g}_{\mu \nu}=\eta_{\mu \nu}, \\
& d S_{4}: \quad \bar{g}_{\mu \nu} d x^{\mu} d x^{\nu}=d t^{2}-e^{2 \sqrt{\Lambda} t}\left(d x_{1}^{2}+d x_{2}^{2}+d x_{3}^{2}\right), \\
& A d S_{4}: \quad \bar{g}_{\mu \nu} d x^{\mu} d x^{\nu}=-d x_{3}^{2}-e^{-2 \sqrt{-\Lambda} x_{3}}\left(d x_{1}^{2}+d x_{2}^{2}-d t^{2}\right) .
\end{aligned}
$$

Accordingly the induced four-dimensional (4D) metric $\bar{g}_{\mu \nu}$ is Minkowski, de Sitter or anti-de Sitter and the corresponding 4D cosmological constant $\Lambda$ has mass dimension equal to 2 . In 
all cases the Ricci-tensor and scalar turn out to be

$$
\begin{aligned}
R_{\mu \nu} & =e^{2 A}\left(4 A^{2}-A^{\prime \prime}-3 \Lambda e^{2 A}\right) \bar{g}_{\mu \nu}, \\
R_{55} & =-4 A^{\prime 2}-4 A^{\prime \prime} \\
R & =20 A^{\prime 2}+8 A^{\prime \prime}-12 \Lambda e^{-2 A}
\end{aligned}
$$

where ${ }^{\prime} \equiv d / d r$. The equation of motion for the scalar field reads

$$
\phi^{\prime \prime}+4 A^{\prime} \phi^{\prime}=\frac{\partial V}{\partial \phi}+\sum_{\alpha} \frac{\partial \lambda_{\alpha}}{\partial \phi} \delta\left(r-r_{\alpha}\right)
$$

and the Einstein equations have the form

$$
\begin{aligned}
A^{\prime \prime}+\Lambda e^{-2 A} & =-\frac{\kappa^{2}}{3} \phi^{\prime 2}-\frac{\kappa^{2}}{3} \sum_{\alpha} \lambda_{\alpha} \delta\left(r-r_{\alpha}\right), \\
A^{\prime 2}-\Lambda e^{-2 A} & =-\frac{\kappa^{2}}{6} V+\frac{\kappa^{2}}{12} \phi^{\prime 2},
\end{aligned}
$$

with $\kappa^{2}=1 /\left(2 M^{3}\right)$. Hereby the localized terms impose the following constraints (assuming a $\mathbb{Z}_{2}$ symmetry across the branes)

$$
\left.A^{\prime}\right|_{r_{\alpha}-\epsilon} ^{r_{\alpha}+\epsilon}=-\frac{\kappa^{2}}{3} \lambda_{\alpha}\left(\phi\left(r_{\alpha}\right)\right),\left.\quad \phi^{\prime}\right|_{r_{\alpha}-\epsilon} ^{r_{\alpha}+\epsilon}=\frac{\partial \lambda_{\alpha}\left(\phi\left(r_{\alpha}\right)\right)}{\partial \phi} .
$$

Using these equations in the action one obtains

$$
S=6 M^{3} \int d^{5} x \sqrt{\left|\operatorname{det} g_{M N}\right|} e^{-2 A} \Lambda .
$$

We would like to emphasize that this relation does not rely on any approximation so far. The key observation is that from an effective four-dimensional point of view the expansion parameter $\Lambda$ has to be related to the value of the radion potential. If the system (8)-(10) allows for several solutions, the corresponding values of $\Lambda$ allow to determine the difference in potential energy of the radion between different configurations. Here we will focus on positive cosmological constants and our aim will be to determine $\Lambda$ with an accuracy that goes beyond the usual weak field assumption $\phi^{2} \ll M^{3}[6]$.

\section{STABILIZATION AND GW MECHANISM}

Solving the system (88)-(10) is for generic scalar potentials a hard task. A possibility to overcome this difficulty is to consider the quadratic Goldberger-Wise (GW) scalar poten- 
tial [6]

$$
\begin{aligned}
V(\phi) & =-\frac{12 M^{3}}{l^{2}}+\frac{1}{2} m^{2} \phi^{2}, \\
\lambda_{\alpha}(\phi) & =\lambda_{\alpha}^{0}+\gamma_{\alpha}\left(\phi-v_{\alpha}\right)^{2} \quad \text { with } \quad \gamma_{\alpha} \rightarrow \infty
\end{aligned}
$$

where $l$ is the AdS length such that $1 / l=k$ is of the order the Planck scale, $\phi$ and $v_{\alpha}$ have mass dimension $3 / 2$ and $\gamma_{\alpha}$ has mass dimension 1 . We assume that these potentials are chosen such that the warping in the metric is close to AdS, meaning that $A(r)$ in (10) is dominated by the bulk vacuum energy

$$
l^{2} \phi^{\prime 2} \ll 24 M^{3}, \quad l^{2} m^{2} \phi^{2} \ll 24 M^{3}, \quad \Lambda \ll l^{-2} .
$$

At leading order in $\phi$ the scalar sector then decouples completely from gravity. The equations of motion for the metric are then given by $\Lambda=0$ and $A^{\prime}=-1 / l$ and the gauge choice $A(0)=0$ leads to the solution

$$
A(r)=-r / l
$$

in the bulk. We assume the UV and IR branes to be located at $r_{1}=0$ and $r_{2}=r_{0}$ respectively.

Finally under the assumptions (15) the solution of the scalar field turns out to be

$$
\begin{aligned}
\phi(r) & =v_{1} e^{k_{-} r}+\left(v_{2}-v_{1} e^{k_{-} r_{0}}\right) \frac{e^{k_{+} r}-e^{k_{-} r}}{e^{k_{+} r_{0}}-e^{k_{-} r_{0}}} \\
l k_{ \pm} & =2 \pm \sqrt{4+m^{2} l^{2}}
\end{aligned}
$$

where $v_{\alpha}$ denote the values of $\phi$ at the UV and IR branes ${ }^{2}$. We focus on negative values ${ }^{3}$ of $k_{-}$and a solution of the hierarchy problem $\left(r_{0} \approx 37 l\right)$ requires

$$
v_{1} \sim v_{2} \sim M^{3 / 2}, \quad 1 \sim e^{k_{-} r_{0}} \ll 10^{16} \sim e^{r_{0} / l}
$$

such that one obtains

$$
\phi(r) \approx v_{1} e^{k_{-} r}+\left(v_{2}-v_{1} e^{k_{-} r_{0}}\right) e^{k_{+}\left(r-r_{0}\right)}
$$

Using this in the action (11) yields

$$
S=l^{-1} \int d^{4} x\left\{l k_{-} v_{1}^{2}-e^{-4 r_{0} / l}\left[\left(4-l k_{-}\right)\left(v_{2}-v_{1} e^{k_{-} r_{0}}\right)^{2}+l k_{-} v_{2}^{2}\right]\right\} .
$$

\footnotetext{
${ }^{2}$ In the brane potentials (14) the linear terms satisfying eqs. (11) and (17) are omitted since the results of our analysis will be independent of them.

${ }^{3}$ Most results in our analysis are easily carried over to positive $k_{-}$.
} 
In ref. [6] this is identified (after a change of sign) with the potential of the radion $V_{G W}$ as

$$
S=-\int d^{4} x V_{G W}\left(r_{0}\right)
$$

and has a minimum at $\xi=\xi_{-}$(i.e. $\left.r_{0}=r_{-}\right)$where

$$
\xi \equiv \frac{v_{1}}{v_{2}} e^{k_{-} r_{0}}, \quad \xi_{-} \equiv \frac{v_{1}}{v_{2}} e^{k_{-} r_{-}}=1+\left|l k_{-}\right|^{1 / 2} / 2-l k_{-} / 4+O\left(\left(l k_{-}\right)^{3 / 2}\right) .
$$

The value of the potential at this minimum is of $\mathcal{O}\left(\mathrm{TeV}^{4}\right)$

$$
V_{G W}\left(r_{-}\right)-V_{G W}(\infty) \simeq l^{-1}\left|l k_{-}\right|^{3 / 2} v_{2}^{2} e^{-4 r_{-} / l} .
$$

However this result is not conclusive. In an effective description of the radion one would like to split the dynamics into four-dimensional gravity and the radion degree of freedom. Hence the radion potential should not only depend on the scalar part of the action but might also receive a contribution from 5D gravity. In the derivation of eq. (223) we neglected contributions to the field $A$ of order $\phi^{2}$ but these terms can potentially change the action and thus the potential seen by the radion. In fact from eq. (12) it is intuitive that these corrections should arise since, in a non-expanding background, $\Lambda=0$, the action should vanish. In particular these additional contributions will change the boundary conditions on the branes and could potentially modify the difference in action between the minimum and the limit $r_{0} \rightarrow \infty$ which is most important for the analysis of the phase transition.

Alternatively the system (8)-(10) for $\Lambda=0$ can be solved by the so-called superpotential method [15, 16]. Its large advantage is that it provides exact solutions since backreactions are automatically taken into account. Starting from a superpotential $W(\phi)$ the equations of motion for the choice

$$
\begin{gathered}
\varepsilon_{\alpha} \lambda_{\alpha}(\phi)=W\left(\phi\left(r_{\alpha}\right)\right)+\frac{\partial W\left(\phi\left(r_{\alpha}\right)\right)}{\partial \phi}\left(\phi-\phi\left(r_{\alpha}\right)\right)+\varepsilon_{\alpha} \gamma_{\alpha}\left(\phi-\phi\left(r_{\alpha}\right)\right)^{2}, \\
V(\phi)=\frac{1}{8}\left(\frac{\partial W}{\partial \phi}\right)^{2}-\frac{\kappa^{2}}{6} W(\phi)^{2}
\end{gathered}
$$

[where $\varepsilon_{1,2} \equiv \pm 1$ refer to the two branes, at $r_{1}=0$ and $r_{2}=r_{0}$ respectively, according to the $\mathbb{Z}_{2}$-orbifold boundary conditions] can be recast in term of the first-order differential equations

$$
\phi^{\prime}=\frac{1}{2} \frac{\partial W}{\partial \phi}, \quad A^{\prime}=-\frac{\kappa^{2}}{6} W
$$


In particular the superpotential of the form

$$
W=\frac{12 M^{3}}{l}+k_{-} \phi^{2}
$$

provides the following scalar potential

$$
V=-\frac{12 M^{3}}{l^{2}}+\frac{\left(k_{-} l\right)^{2}-4 k_{-} l}{2 l^{2}} \phi^{2}-\frac{\left(k_{-} l\right)^{2} \kappa^{2}}{6 l^{2}} \phi^{4} .
$$

For the aim of estimating $\Lambda$ at order $\phi^{2}$, eq. (29) coincides with the potential (13) after imposing eq. (18) and requiring $\phi^{2} \ll M^{3}$. The corresponding solutions to the equations of motion read [15, 16]

$$
\begin{aligned}
& A=-\frac{r}{l}-\frac{1}{6} v_{1}^{2} e^{2 k_{-} r} \\
& \phi=v_{1} e^{k_{-} r}
\end{aligned}
$$

Consequently once one chooses $v_{2}$ the brane distance is fixed by

$$
\xi_{-}=\frac{v_{1}}{v_{2}} e^{k_{-} r_{-}}=1
$$

which differs from eq. (23) . Furthermore notice that this solution is based on the requirement $\Lambda=0$, which means that the action at its extremum $\xi=\xi_{-}$vanishes $[c f$. eq. (12)]. On the other hand in the limit $r_{0} \rightarrow \infty$ the $\phi$ profile (31), which matches with (17), is still a solution of the equations of motion with $\Lambda=0$ and thus the radion potential also approaches asymptotically a vanishing cosmological constant. Hence the superpotential method shows that backreactions can have an important impact on the radion potential.

\section{RADION POTENTIAL INCLUDING BACKREACTIONS}

The superpotential method can also be generalized to non-vanishing cosmological constant [16] and in principle every solution to the equations (8)-(10) can be exactly derived from some superpotential. Nevertheless it is not of much use in determining the radion potential since, for fixed scalar bulk and brane potentials, it cannot be used to find several solutions corresponding to different brane separations ${ }^{4}$. Understanding the structure of the radion potential (partially) including backreactions is the aim of this section.

4 Using the superpotential method a change in the brane separation would lead, according to (25), to a change in the brane potentials. 
For an arbitrary brane separation the system (8)-(9) does not always have a solution. There are three integration constants, the brane separation $r_{0}$ and the parameter $\Lambda$ and four constraints on the branes (11). However only the combination $\Lambda e^{2 A}$ enters in the equations and one integration constant can be eliminated. It can be used to choose e.g. $A(0)=0$ or $|\Lambda|=1$. In summary we have four constants to be fixed by four boundary conditions, so generically one expects a unique solution for given bulk and brane potentials. This is not too surprising since one would expect that the system does not allow for a time-independent solution (up to Hubble expansion) for the radion field when it is not located at an extremum of the potential.

Naively one would like to determine the action for several brane separations and identify it with the negative potential seen by the radion. This is basically the procedure followed by Goldberger and Wise in a fixed gravitational background. However there are two objections to calculating the radion potential in this way if backreactions are included. First, solutions to the Einstein equations do not constitute an extremum of the Einstein-Hilbert action, so the action of the gravitational part should have no physical significance. This problem can be easily overcome by including the Gibbons-Hawking term in the action [17, 18] as we will see. Second, the system of equations (8)-(10) only allows for brane separations that correspond to extrema in the radion potential. One way of avoiding this latter problem would be to solve all equations including a time-dependence and this program is followed in the vicinity of the static solution in ref. [3], though without any stabilization mechanism for the radion.

In the present work we will present an alternative and simplified treatment which includes the bulk potential. We first determine the action and the radion mass in the extrema of the radion potential and, after computing the kinetic term, we use this information to get a reliable fit to the whole potential. We finally check that the results of our effective fourdimensional theory are consistent with the ones of ref. [3].

We will focus on the particular case of the bulk and brane potentials (13)-(14) constrained by the bounds (15) and with solution (20). However the rationale we follow can be used to analyze the radion potential also in other scenarios. 


\section{A. Cosmological constant and position of the extrema}

The values of the action at its extrema are related to the expansion parameter $\Lambda$ at those points according to (12). We will determine this parameter in this section. A basic ingredient for our analysis is the scalar solution (20) which was obtained under the constraints (15). Besides we make use of stiff scalar potentials on the branes. In this case the scalar field is fixed to the values $v_{\alpha}$ on the branes and eq. (10) in combination with the boundary conditions (11) reads

$$
\Lambda e^{-2 A\left(r_{\alpha}\right)}+\left.\frac{\kappa^{2}}{12} \phi^{\prime 2}\right|_{r=r_{\alpha}}=\frac{\kappa^{4}}{36} \lambda_{\alpha}^{2}\left(v_{\alpha}\right)+\frac{\kappa^{2}}{6} V\left(v_{\alpha}\right) .
$$

On the basis of this equation it is possible to accurately determine the expansion parameter $\Lambda$ including ${ }^{5}$ backreactions. Notice that due to the presence of stiff brane potentials only the left-hand side depends on the brane separation, once the model parameters are fixed, while the right-hand side can be arbitrarily chosen due to the brane potentials. In the following we will first discuss the case where the brane potential is tuned to reproduce the results obtained with the superpotential method for the choice

$$
\varepsilon_{\alpha} \lambda_{\alpha}^{0}=\frac{12 M^{3}}{l}+k_{-} v_{\alpha}^{2}
$$

and we will subsequently detune it to obtain cosmologically more realistic potentials as the one deduced by Goldberger and Wise.

\section{Tuned case}

Let us consider eq. (33) evaluated for the two branes at $r \in\left\{0, r_{0}\right\}$. For the brane potentials $\lambda_{\alpha}^{0}$ used in the superpotential method these two equations read

$$
\begin{aligned}
24 M^{3} \Lambda_{1} & =v_{1}^{2} k_{-}^{2}-\left(v_{1} k_{-}+k_{+}\left(v_{2}-v_{1} e^{k_{-} r_{0}}\right) e^{-k_{+} r_{0}}\right)^{2}, \\
24 M^{3} \Lambda_{2} e^{2 r_{0}} & =v_{2}^{2} k_{-}^{2}-\left(v_{1} k_{-} e^{k_{-} r_{0}}+k_{+}\left(v_{2}-v_{1} e^{k_{-} r_{0}}\right)\right)^{2},
\end{aligned}
$$

where we have made use of the equality

$$
m^{2}=k_{-}^{2}-4 k_{-} / l
$$

\footnotetext{
${ }^{5}$ Subject to the constraints in (15).
} 
Owing to the choice of brane potentials two solutions with vanishing expansion parameter, $\Lambda=0$, are given by $r_{0} \rightarrow \infty$ and $r_{0}=r_{-}$with

$$
\frac{v_{1}}{v_{2}} e^{k_{-} r_{-}}=1
$$

as obtained by the superpotential method. Nevertheless there is an additional solution with a larger brane separation and a positive cosmological constant that leads to a positive Einstein-Hilbert action according to eq. (12). As an example we show in fig. 11 the functions $\Lambda_{1,2}$ versus $r_{0}$ corresponding to a given set of parameters.

An analytic estimate for the second solution can be obtained as follows. Notice that the function $\Lambda_{1}$ is small compared to $\Lambda_{2}$ due to the factor $e^{-k_{+} r_{0}}$ and

$$
24 M^{3} \Lambda_{1} \approx-2 k_{-} k_{+} v_{1}\left(v_{2}-v_{1} e^{k_{-} r_{0}}\right) e^{-k_{+} r_{0}}
$$

Hence the values of $r_{0}$ for the solutions to $\Lambda_{1}=\Lambda_{2}$ are close to the zeros of $\Lambda_{2}$ that are given by

$$
\xi_{\mp} \equiv \frac{v_{1}}{v_{2}} e^{k_{-} r_{\mp}}=\frac{k_{+} \mp k_{-}}{k_{+}-k_{-}} .
$$

The first solution is just the usual solution obtained by the superpotential method

$$
r_{-}=\frac{1}{k_{-}} \log \frac{v_{2}}{v_{1}}
$$

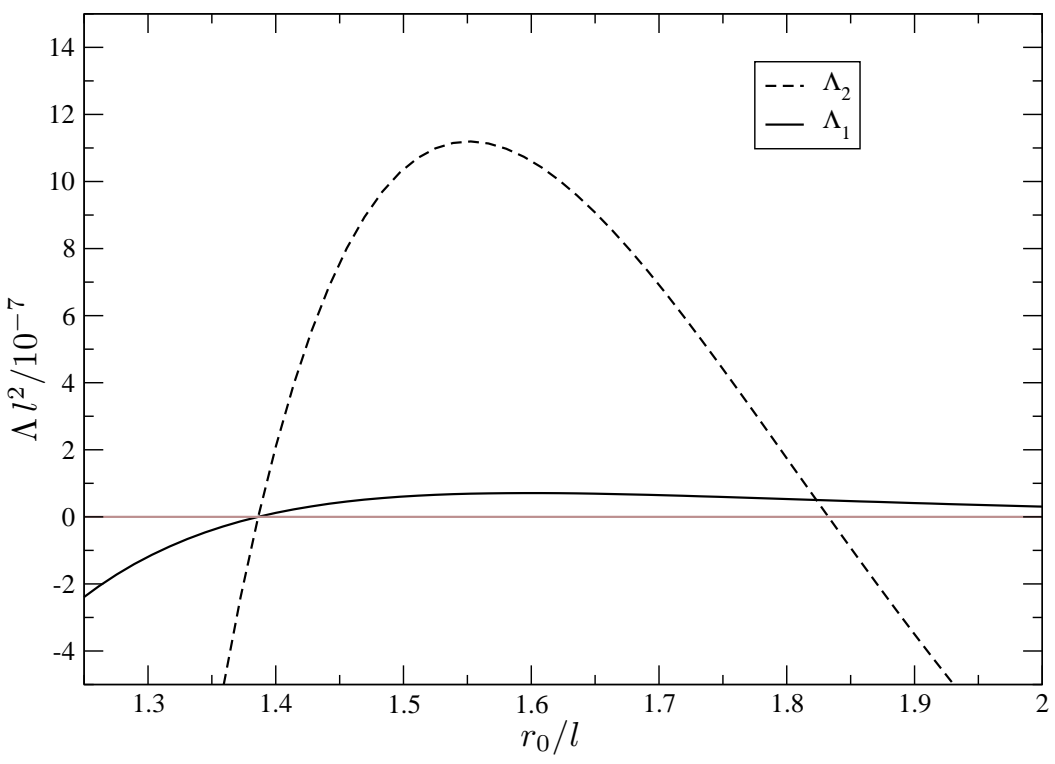

FIG. 1: The functions $\Lambda_{1,2}$ leading to the two different solutions of the equations of motion. The parameters used are $k_{-} l=0.5, v_{1}=0.1 M^{3 / 2}, v_{2}=0.05 M^{3 / 2}$ and $l=M^{-1}$ which corresponds to $m^{2} l^{2}=2.25$. The values are chosen for illustrative purposes and do not lead to a realistic hierarchy. 
while the other solution is given by

$$
r_{+}=r_{-}+\frac{1}{k_{-}} \log \frac{k_{+}+k_{-}}{k_{+}-k_{-}} \approx r_{-}+\frac{2}{k_{+}} \approx r_{-}+\frac{l}{2} .
$$

Using this value in eq. (38) gives for $\Lambda$ the value

$$
\Lambda_{+} \approx \frac{k_{-}^{2}}{6} \frac{v_{1} v_{2}}{M^{3}} e^{-4 r_{+} / l}
$$

The expansion parameter $\Lambda_{+}$is in this case additionally suppressed compared to the energy scale involved in the hierarchy problem and one might attempt to use this fact to solve, or at least to alleviate, the cosmological constant problem in a similar way to the proposals in ref. [19]. However and not unexpectedly, as we will find in a later section, this solution does correspond to a maximum in the radion potential and is not stable.

Let us compare this result with the findings from the superpotential method. Since backreactions are taken into account to order $\phi^{2}$ the action and the brane separation $r_{-}$should agree to this order and they indeed precisely do. Notice that the expansion parameter $\Lambda$ for an infinitely large brane separation also vanishes in agreement with the superpotential method. One can thus infer that the radion potential has two degenerate minima at $r_{0}=\left\{r_{-}, \infty\right\}$ separated by a maximum at $r_{+}{ }^{6}$. Consequently a realistic phase transition proceeding from $\infty$ to $r_{-}$requires some detuning away from the scenario with a superpotential, e.g. by changing the brane potentials which is the next step of our analysis.

\section{Detuned case}

Using the brane potentials of the tuned case above does not allow a realistic phase transition. Since the radion potential is degenerate at $r_{0}=r_{-}$and $r_{0} \rightarrow \infty$ the system would be stuck in the high temperature phase. For a realistic model we should modify these two configurations by some detuning of the UV and IR brane tensions. Following the same steps leading to eqs. (35) the new boundary conditions for $A^{\prime}$ in eq. (11) lead to

$$
\begin{aligned}
24 M^{3} \Lambda_{1} & =v_{1}^{2} k_{-}^{2}-\left(v_{1} k_{-}+k_{+}\left(v_{2}-v_{1} e^{k_{-} r_{0}}\right) e^{-k_{+} r_{0}}\right)^{2}+c_{1} v_{1}^{2}, \\
24 M^{3} \Lambda_{2} e^{2 r_{0}} & =v_{2}^{2} k_{-}^{2}-\left(v_{1} k_{-} e^{k_{-} r_{0}}+k_{+}\left(v_{2}-v_{1} e^{k_{-} r_{0}}\right)\right)^{2}+c_{2} v_{2}^{2}
\end{aligned}
$$

where we have parameterized the detuning by $c_{\alpha} v_{\alpha}^{2} \equiv \frac{2}{3}\left(\lambda_{\alpha}^{2}-\lambda_{\alpha}^{0^{2}}\right)$.

\footnotetext{
${ }^{6}$ A more detailed explanation why this guess is correct will be provided in the next subsection.
} 
We first enforce that today's observed expansion is small. If the minimum of the potential has a vanishing expansion parameter $\Lambda$, according to eq. (44) it is given by

$$
\xi_{-}=\frac{v_{1}}{v_{2}} e^{k_{-} r_{-}}=\frac{k_{+}+\sqrt{k_{-}^{2}+c_{2}}}{k_{+}-k_{-}},
$$

which depends on the free parameter $c_{2}$. For $\Lambda=0$ eq. (43) reads

$$
0=24 M^{3} \Lambda_{1} \approx 2 v_{1} v_{2} k_{+} k_{-}(\xi-1) e^{-k_{+} r}+c_{1} v_{1}^{2}
$$

which together with the position of the minimum $\xi_{-}$in eq. (45) fixes the value of $c_{1}$ as

$$
c_{1} v_{1}^{2}=2 v_{1} v_{2} k_{+}\left|k_{-}\right|\left(\xi_{-}-1\right) e^{-k_{+} r_{-}} .
$$

This choice is the fine-tuning that is needed to solve the cosmological constant problem and it is in general present in RS-type models.

Once one fixes $\xi_{-}$(and correspondingly $c_{1}$ and $c_{2}$ ) the system (43)-(44) has a second solution $\xi_{+}$that fulfills

$$
\begin{aligned}
& k_{-}^{2}+c_{2}-\left(k_{-} \xi_{+}+k_{+}\left(1-\xi_{+}\right)\right)^{2} \approx c_{1} \frac{v_{1}^{2}}{v_{2}^{2}} e^{2 r_{+} / l} \\
& =2 \frac{v_{1}}{v_{2}} k_{+}\left|k_{-}\right|\left(\xi_{-}-1\right) e^{-k_{+} r_{-}+2 r_{+} / l} .
\end{aligned}
$$

As long as $c_{2}$ is small the right-hand side can be neglected and the position of $\xi_{+}$is, in a similar way to the minimum $\xi_{-}$, given by

$$
\xi_{+}=\frac{v_{1}}{v_{2}} e^{k_{-} r_{+}}=\frac{k_{+}-\sqrt{k_{-}^{2}+c_{2}}}{k_{+}-k_{-}}=\frac{2 k_{+}}{k_{+}-k_{-}}-\xi_{-} .
$$

On the other hand if $c_{2}$ surpasses $k_{+}^{2}-k_{-}^{2}$ the left-hand side is positive for $\xi_{+}>0$. In this case, the right-hand side has to become comparatively large what happens at $r_{+} \approx \frac{k_{+} l}{2} r_{-} \approx 2 r_{-}$ which implies $\xi_{+} \approx \frac{v_{2}}{v_{1}} \xi_{-}^{2}<\xi_{-}$for $k_{-}<0$. Comparison shows that sizeable deviations from (49) are hence not expected for

$$
\xi_{-} \lesssim-\frac{v_{1}}{2 v_{2}}+\sqrt{\frac{v_{1}^{2}}{4 v_{2}^{2}}+\frac{2 v_{1}}{v_{2}}}
$$

It turns out that the parameter space that violates this bound is less interesting in the sense that it leads generally to rather strong phase transitions. In this way we will mostly be concerned with the region in parameter space where (49) is a good approximation. 
Plugging eq. (47) in (43) one finds the expansion parameter at the maximum $\xi_{+}$to be

$$
\Lambda_{+} \approx \frac{v_{1} v_{2}}{12 M^{3}} k_{+}\left|k_{-}\right|\left(\left(\xi_{-}-1\right) e^{-k_{+} r_{-}}+\left(1-\xi_{+}\right) e^{-k_{+} r_{+}}\right) .
$$

If $\xi_{-}$is not too close to unity the first contribution dominates this expression because $r_{+}>r_{-}$. On the other hand the cosmological constant in the limit $\xi \rightarrow 0\left(r_{0} \rightarrow \infty\right)$ is given by this first contribution

$$
\Lambda_{\infty} \approx \frac{v_{1} v_{2}}{12 M^{3}} k_{+}\left|k_{-}\right|\left(\xi_{-}-1\right) e^{-k_{+} r_{-}} .
$$

as it can be easily deduced from eqs. (43) and (47). The radion potential as a function of $\xi$ is hence very flat between $\xi=0$ and $\xi=\xi_{+}$and then quickly drops to zero at $\xi=\xi_{-}$. In a subsequent subsection we will provide a parametrization that interpolates between these extremal values.

We will also demand that this construction solves the hierarchy problem, i.e. $r_{-} \approx 37 l$, and thus for fixed ratio $v_{1} / v_{2}$ the parameter $m^{2}$ (and hence $k_{-}$) should be accordingly chosen while we treat $\xi_{-}$(or $r_{-}$) as a free parameter. In principle $c_{2}$ can always be chosen such that one obtains for $\xi_{-}$an arbitrary value in the interval $[1, \infty]$ (with $\Lambda_{\infty} \geq 0$ ) but we will assume $\xi_{-}$to be smaller than $v_{1} / v_{2}$ in order to obtain a negative $k_{-}$

$$
k_{-}=\frac{1}{r_{-}} \log \left(\frac{v_{2}}{v_{1}} \xi_{-}\right) \text {. }
$$

About the approximations we employed, notice that we used (13) as scalar potential and nowhere the assumption $\phi^{2} \ll M^{3}$ that needs to be fulfilled in order to make contact with the superpotential method. The sole constraint for the applicability of our results so far is (15). This leads to the inequalities [using (201)]

$$
m^{2} l^{2} \phi^{2} \lesssim m^{2} l^{2} v_{1}^{2} \approx-4 l k_{-} v_{1}^{2} \ll 24 M^{3}
$$

and

$$
l^{2} \phi^{\prime 2}=v_{2}^{2}\left(l k_{-} \xi+l k_{+}(1-\xi)\right)^{2}<v_{2}^{2}\left(l k_{-} \xi_{-}+l k_{+}\left(1-\xi_{-}\right)\right)^{2} \ll 24 M^{3} .
$$

Depending on the parameters these constraints can be much weaker than those employed in the literature to ensure smallness of backreactions [6, 10] namely $\phi^{2} \ll M^{3}$. However, they still guarantee that the deformation of the CFT induced by the operators corresponding to the bulk scalar can be treated perturbatively at all scales up to the $4 \mathrm{D}$ Planck scale ${ }^{7}$.

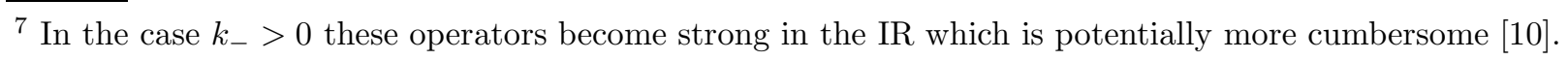


Notice that even if the conditions (54) and (55) are fulfilled (which ensures that the relative error in $A^{\prime}$ is small) there still can be sizeable (cumulative) deviations in $e^{A}$ since $A\left(r_{0}\right) \approx 37$. Nevertheless this will not affect the equations of motion of $\phi$ that only depend on $A^{\prime}$ and not on the exponential warp factor. Hence the expansion parameter deduced from the equations (43)-(44) is reliable. Still the detuning parameters need in principle (small) corrections to reproduce the correct hierarchy. In the following we call the regime where our constraints are saturated and our approximation becomes unreliable the regime of large backreactions.

Let us compare these findings with the results from the superpotential method and those in the GW framework. The GW potential can be written as

$$
V_{G W}=l^{-1} v_{2}^{2}\left(\frac{v_{1}}{v_{2}} \xi\right)^{-4 /\left(l k_{-}\right)}\left[\left(4-l k_{-}\right)(1-\xi)^{2}+l k_{-}\right]+\text {const }
$$

and the extrema fulfill

$$
\frac{4}{l k_{-} \xi_{ \pm}}=\frac{2\left(4-l k_{-}\right)\left(1-\xi_{ \pm}\right)}{\left(4-l k_{-}\right)\left(1-\xi_{ \pm}\right)^{2}+l k_{-}} .
$$

This leads to a quadratic equation for $\xi_{ \pm}$with solutions that are of the form

$$
\xi_{ \pm}=\frac{k_{+} \mp \sqrt{k_{-}^{2}+c_{2}}}{k_{+}-k_{-}}
$$

for an appropriate $c_{2}$. This coincides with the positions of the extrema in the detuned case found in (45) and (49) under the assumption (50). The value of the potential in the extrema is given by

$$
V_{G W}\left(r_{ \pm}\right)-V_{G W}(\infty)=2 v_{2}^{2} l^{-1} e^{-4 r_{ \pm} / l}\left(l k_{-}\right) \xi_{ \pm}\left(1-\xi_{ \pm}\right)
$$

which is proportional to the difference in expansion parameters $\left(\Lambda_{ \pm}-\Lambda_{\infty}\right)$. The value of the radion potential can indeed be inferred from the expansion parameter $\Lambda$ as we will see in section IVC. In conclusion our results agree [at least to order $\mathcal{O}\left(\phi^{2}\right)$ ] with the superpotential method for the choice

$$
\xi_{-}=1 \quad(\text { superpotential method })
$$

while the GW potential (up to a constant piece) is reproduced for the choice

$$
\xi_{-} \simeq 1+\left|l k_{-}\right|^{1 / 2} / 2-l k_{-} / 4 \quad \text { (Goldberger-Wise) }
$$

under the assumption (50). Hence our approach unifies the results obtained both in the GW and superpotential approaches. 
Finally some comments on the role of the original GW potential are in order. In our framework the potential obtained by Goldberger and Wise merely corresponds to a specific choice for the parameter $\xi_{-}$. From our point of view there is no special significance to this choice and it just results from imposing initially the same tension on the two branes when the backreactions are ignored. Once the behavior of the scalar field is determined one would be forced to adjust these brane tensions in order to cancel the contributions from the backreactions and to obtain a vanishing expansion parameter at the minimum (as already mentioned in ref. [6]). Hence this parameter choice is in no way a distinguished one. In particular the fact that the potential difference (24) scales with $\left(l k_{-}\right)^{3 / 2}$ results from the peculiar choice in (61) (or equivalently for the brane potentials). This scaling is also reflected in the radion mass as we next discuss.

\section{B. The radion mass}

In this subsection we will determine the radion mass in the detuned case along the lines of ref. [15] while the decoupling of the linearized Einstein equations is demonstrated in appendix A. It turns out that in the case of an expanding Universe the impact of the expansion parameter $\Lambda$ can be absorbed in the radion mass parameter

$$
\hat{m}_{r a d}^{2}=m_{r a d}^{2}+6 \Lambda
$$

However the contribution from $\Lambda$ is anyway negligible (being smaller by a factor $e^{2 A}$ ) leading to the equation [see eq. (A15)]

$$
\hat{F}^{\prime \prime}+2 A^{\prime \prime} \hat{F}-2 A^{\prime} \hat{F}^{\prime}-2 \frac{\phi_{0}^{\prime \prime}}{\phi_{0}^{\prime}} \hat{F}^{\prime}=-m_{r a d}^{2} e^{-2 A} \hat{F},
$$

which can be solved to obtain $m_{\text {rad }}$. If backreaction is neglected, $A^{\prime \prime}=0$, this allows for a solution with $\hat{F}=1$ and $m_{\text {rad }}^{2}=0$. For small backreaction, $\hat{F}=1+f$, this system of equations can be linearized as

$$
\begin{aligned}
f^{\prime \prime}-2 A^{\prime} f^{\prime}-2 \frac{\phi_{0}^{\prime \prime}}{\phi_{0}^{\prime}} f^{\prime} & =-2 A^{\prime \prime}-m_{r a d}^{2} e^{-2 A} \\
& =\frac{2 \kappa^{2}}{3} \phi_{0}^{\prime 2}-m_{r a d}^{2} e^{-2 A}
\end{aligned}
$$

while the boundary conditions in the limit $\gamma_{\alpha} \rightarrow \infty$ read $f^{\prime}(0)=f^{\prime}\left(r_{0}\right)=0$. The main difference with respect to the standard solution presented in ref. [3] comes from the factor $\phi_{0}^{\prime \prime} / \phi_{0}^{\prime}$ that deviates from $k_{-}$close to the TeV brane. 
The solution reads

$$
f^{\prime}(r)=\phi^{\prime 2}(r) e^{2 A(r)} \int_{0}^{r} d x\left(\frac{2 \kappa^{2}}{3} e^{-2 A(x)}-\frac{m_{r a d}^{2}}{\phi^{\prime 2}(x)} e^{-4 A}\right),
$$

that by construction fulfills the boundary condition $f^{\prime}(0)=0$. At this point we will neglect backreactions in $A$ that would lead to corrections of order $O\left(\phi^{4}\right)$ on the radion mass ${ }^{8}$. Then the constraint $f^{\prime}\left(r_{0}\right)=0$ determines the mass by the equation

$$
m_{\text {rad }}^{2} \int_{0}^{r_{0}} d x \frac{e^{4 x / l}}{\phi^{\prime 2}(x)}=l \frac{\kappa^{2}}{3} e^{2 r_{0} / l} .
$$

For the scalar field solution in the limit of small backreactions (15), which can be written

$$
\phi^{\prime}(r)=v_{1} k_{-} e^{k_{-} r}\left(1-\tilde{q} e^{\left(k_{+}-k_{-}\right)\left(r-r_{0}\right)}\right), \quad \tilde{q}=k_{+} / k_{-}\left(1-\frac{1}{\xi}\right)
$$

the above integral becomes (using $l k_{+}=4-l k_{-}$)

$$
\int_{0}^{r_{0}} d x \frac{e^{4 x / l}}{\phi^{\prime 2}(x)} \approx \frac{l e^{\left(4-2 l k_{-}\right) r_{0} / l}}{4 v_{1}^{2} k_{-}^{2}(1-\tilde{q})} .
$$

For $\tilde{q}>1$ the integrand actually contains a pole. However a more sophisticated analysis shows that the solution (65) is still regular due to the pre-factor $\phi^{\prime 2}(r)$ and that the naive integration is justified. Therefore one concludes that the radion mass is

$$
\begin{aligned}
m_{\text {rad }}^{2} & =\frac{2}{3} k_{-}^{2}(1-\tilde{q}) e^{\left(-2+2 l k_{-}\right) r_{0} / l} \frac{v_{1}^{2}}{M^{3}} \\
& \approx \frac{8}{3 l^{2}} l k_{-}\left(1-\xi+\xi \frac{k_{-}}{k_{+}}\right) \xi e^{-2 r_{0} / l} \frac{v_{2}^{2}}{M^{3}} .
\end{aligned}
$$

Observe that in the case without detuning (corresponding to a scenario that can be treated with the superpotential method)

$$
\xi_{ \pm}=\frac{k_{+} \pm k_{-}}{k_{+}-k_{-}}
$$

the masses turn out to be

$$
m_{r a d, \pm}^{2} \approx \mp \frac{2}{3 l^{2}}\left(l k_{-}\right)^{2} e^{-2 r_{ \pm} / l} \frac{v_{2}^{2}}{M^{3}} \quad(\text { superpotential })
$$

\footnotetext{
${ }^{8}$ When approaching the region of large backreactions one would have to take the above mentioned cumulative effect in the warp factor into account and the final radion mass would involve the corrected warp factor instead of the plain one. Notice that the determination of the radion mass only serves as an additional check on our radion effective action and the tunneling analysis of the radion is generally not affected by this subtlety.
} 
which agrees with the physical radion mass $m_{\text {rad }}^{2}$ found in [15] and also with the interpretation that $\xi_{-}$denotes a minimum of the potential while $\xi_{+}$is a maximum. Moreover in the case of GW, see eq. (61), the mass is

$$
\left.m_{\text {rad }}^{2} \approx \frac{4}{3 l^{2}}\left|l k_{-}\right|^{3 / 2} e^{-2 r_{0} / l} \frac{v_{2}^{2}}{M^{3}} \quad \text { (Goldberger-Wise }\right),
$$

which indeed scales with $\left|l k_{-}\right|^{3 / 2}$ as expected from the GW potential in eq. (13).

Notice that the radion mass is slightly below the $\mathrm{TeV}$ scale what is essential for an effective four-dimensional description. To compare the radion mass obtained here with the one from the radion potential involves the kinetic term which is the topic of the next subsection.

\section{Kinetic term}

So far we have only discussed the occurring expansion parameter $\Lambda$ for the different solutions of the equations of motion. The main motivation was the relation (12) that implies that the action is proportional to the expansion parameter. In the current subsection we will make contact between the five-dimensional system and the effective action of the radion.

Let us start with the kinetic term of the radion. It was derived in several ways [3, 20, 21] and here we briefly review the calculation of ref. [15]. We will use the metric (A1) but will neglect the contributions from the scalar field. These effects are suppressed by a factor $\left(l k_{-}\right)^{2} v_{1}^{2} / M^{3}[15]$ which is small under the constraints (55). The Einstein equations are then solved by the radion Ansatz $F(x, r)=e^{2 r / l} R(x)$ and the Einstein-Hilbert action contains a kinetic term of the form

$$
K_{\text {rad }}=6 M^{3} l \int d^{4} x \sqrt{\left|\operatorname{det} \bar{g}_{\mu \nu}\right|}\left(e^{2 r_{0} / l}-1\right)(\partial R)^{2} .
$$

The correct normalization is obtained by the observation that in this background the geodesic distance of the branes is given by

$$
r(x)=\int_{0}^{r_{0}} d r\left(1-2 e^{2 r / l} R(x)\right)=r_{0}-l e^{2 r_{0} / l} R(x),
$$

from which $(\partial R)^{2}=l^{-2} e^{-4 r_{0} / l}(\partial r)^{2} \simeq l^{-2} e^{-4 r / l}(\partial r)^{2}$. Hence $K_{\text {rad }}$ becomes

$$
\begin{aligned}
K_{\text {rad }} & =12 M^{3} l^{-1} \int d^{4} x \sqrt{\left|\operatorname{det} \bar{g}_{\mu \nu}\right|} \frac{1}{2}(\partial r)^{2} e^{-2 r / l} \\
& =12(M l)^{3} \int d^{4} x \sqrt{\left|\operatorname{det} \bar{g}_{\mu \nu}\right|} \frac{1}{2}(\partial \mu)^{2}
\end{aligned}
$$


with the notation $\mu=l^{-1} e^{-r / l}$. We do not absorb the pre-factor $12(M L)^{3}$ in the definition of $\mu$ because it will prove to be useful in the analysis of the phase transition ${ }^{9}$.

In ref. [6] the action resulting from the bulk scalar was right away interpreted as the negative potential seen by the radion. In the present case this could hardly be true since the action is positive for an expanding Universe. We better expect this to correspond to a maximum and not to a minimum of the potential, since on the other hand the radion mass is negative in this situation. Moreover an expanding Universe without any bulk scalar would also have a non-vanishing action, even though the radion does not see any potential in this case.

In order to obtain the potential seen by the radion we must separate the action into the contributions from the expansion and that from the radion interacting with the bulk scalar. The contribution to the action from the expansion is according to (17) given by

$$
S_{\text {exp }}=12 M^{3} \int d^{5} x \sqrt{\left|\operatorname{det} g_{M N}\right|} e^{-2 A} \Lambda
$$

and, considering the action provided by (12), it results in a radion action

$$
S_{r a d}=-6 M^{3} \int d^{5} x \sqrt{\left|\operatorname{det} g_{M N}\right|} e^{-2 A} \Lambda
$$

which must arise from the value of the radion potential at its extrema.

As mentioned before an additional problem arises from the fact that the solution is not an extremum of the action in general relativity. To overcome this problem one can confine the system to a box, $t \in[-T, T]$, and add the so-called Gibbons-Hawking [18] term to the action 10

$$
S_{G H}=\int_{\partial M} 2 K
$$

where $K$ denotes the extrinsic curvature and $\partial M$ is the boundary of the space-time manifold. Using the metric given in (2) one can evaluate the Gibbons-Hawking term [22]

$$
S_{G H}=-18 M^{3} \int d^{5} x \sqrt{\left|\operatorname{det} g_{M N}\right|} e^{-2 A} \Lambda
$$

\footnotetext{
${ }^{9}$ Notice that this kinetic term was obtained by decoupling the Einstein equations and the result is a factor of 2 smaller than the one from the more naive approach derived in [6, 10] and subsequently used in the analysis of the phase transition in $10,11,13]$.

10 Inclusion of this term is actually not relevant since the radion potential could be inferred from (77), but it will elucidate the comparison with ref. [3] later on.
} 
and the total action is given by

$$
S_{t o t}=S_{e x p}+S_{r a d}+S_{G H}=-12 M^{3} \int d^{5} x \sqrt{\left|\operatorname{det} g_{M N}\right|} e^{-2 A} \Lambda .
$$

After integration over the fifth dimension the effective action for the radion hence reads ${ }^{11}$

$$
\begin{aligned}
S_{\text {eff }}= & 12(M l)^{3} \int d^{4} x \sqrt{\left|\operatorname{det} \bar{g}_{\mu \nu}\right|}\left(\frac{1}{2}(\partial \mu)^{2}-V_{\text {rad }}(\mu)\right) \\
& +M_{P}^{2} \int d^{4} x \sqrt{\left|\operatorname{det} \bar{g}_{\mu \nu}\right|} R_{(4)}+S_{G H},
\end{aligned}
$$

where we defined the four-dimensional Planck mass, $\left(M_{P} l\right)^{2}=(M l)^{3}$, and identified the radion potential in its extrema as

$$
V_{\text {rad }}\left(r_{ \pm}\right)=l^{-2} \Lambda_{ \pm} / 2
$$

This is the essential relation that we use to connect the value of the radion potential to the expansion around its extrema.

Let us compare this result with the one obtained in ref. [3]. There an effective action was obtained by perturbing the background metric but without taking a stabilizing mechanism into account. Their result reads in our notation ( $a$ is the scale factor)

$$
\begin{aligned}
S_{e f f} & \propto \int d t a^{3}\left(\frac{1}{2} \dot{r}^{2} e^{-2 r / l} l^{-2}+\frac{1}{2}\left(\frac{\ddot{a}}{a}+\left(\frac{\dot{a}}{a}\right)^{2}\right)-V_{\text {rad }}(r)\right)+S_{G H} \\
& =\int d t a^{3}\left(\frac{1}{2} \dot{r}^{2} e^{-2 r / l} l^{-2}-\frac{1}{2}\left(\frac{\dot{a}}{a}\right)^{2}-V_{\text {rad }}(r)\right) .
\end{aligned}
$$

The last equality is obtained by partial integration which cancels the Gibbons-Hawking term at a time-like boundary as discussed above. The radion potential $V_{\text {rad }}(r)$ was not specified in [3] but comparison with our result (81) confirms that the radion potential and the expansion (including the Gibbons-Hawking term) contribute equally to the action in the extrema of the radion potential.

\section{Interpolating potential}

In the following we will approximate the effective potential seen by the radion. The equations of motion have only stationary (up to the Hubble expansion) solutions in the

${ }^{11}$ Note that we integrate over the orbifold $S_{1} / \mathbb{Z}_{2}$ and hence twice over the bulk as it is customary in the literature on RS models. 
three extremal situations $r \in\left\{r_{-}, r_{+}, \infty\right\}$ or $\left.\xi \in\left\{\xi_{-}, \xi_{+}, 0\right\}\right]$ and we use these three values to provide a fit to the potential. The positions of the extrema of the radion potential are given by eqs. (45) and (48). We parametrize the potential by

$$
V_{\text {rad }}^{\prime}(\xi) \propto \xi^{\omega-1}\left(\xi-\xi_{-}\right)\left(\xi-\xi_{+}\right)
$$

and integration yields

$$
V_{\text {rad }}(\xi) \propto \xi^{\omega}\left(\frac{\xi^{2}}{\omega+2}-\frac{\xi\left(\xi_{-}+\xi_{+}\right)}{\omega+1}+\frac{\xi_{-} \xi_{+}}{\omega}\right)
$$

where the integration constant is fixed to zero by $V_{\text {rad }}(0)=0$. The parameters $\omega$ and the pre-factor can be adjusted in order to reproduce $V_{\text {rad }}\left(\xi_{ \pm}\right)$given by eqs. (51), (152) and (82). This corresponds to the expression

$$
V_{\text {rad }}(\xi) l^{4}=\lambda e^{-k_{+} r} P(\xi),
$$

with

$$
\lambda=\frac{1}{24} \frac{v_{1} v_{2}}{M^{3}} l k_{-} l k_{+}
$$

and

$$
P=\frac{1-\xi_{-}}{\xi_{-}}\left(\frac{\xi}{\xi_{-}}\right)^{\omega+k_{+} / k_{-}} \frac{\omega(\omega+1) \xi^{2}-\omega(\omega+2)\left(\xi_{-}+\xi_{+}\right) \xi+(\omega+1)(\omega+2) \xi_{-} \xi_{+}}{\omega\left(\xi_{-}-\xi_{+}\right)-2 \xi_{+}} .
$$

The function $P(\xi)$ is normalized to $P\left(\xi_{-}\right)=\left(\xi_{-}-1\right)$ and $\omega$ is determined by $P\left(\xi_{+}\right)=\left(\xi_{+}-1\right)$, which yields

$$
\left(\frac{\xi_{+}}{\xi_{-}}\right)^{\omega+k_{+} / k_{-}+1} \frac{\omega\left(\xi_{+}-\xi_{-}\right)-2 \xi_{-}}{\omega\left(\xi_{-}-\xi_{+}\right)-2 \xi_{+}}=\frac{1-\xi_{+}}{1-\xi_{-}}
$$

and hence $\omega \approx-k_{+} / k_{-}$.

Another ingredient useful for the fit is the radion mass at the extrema where the second derivative of $V_{\text {rad }}$ turns out to be

$$
\xi^{2} \frac{d^{2}}{d \xi^{2}} V_{r a d}\left(\xi_{ \pm}\right)=\lambda \omega^{2} e^{-k_{+} r} \frac{\left(1-\xi_{ \pm}\right)\left(\xi_{-}-\xi_{+}\right)}{\left(\xi_{-}-\xi_{+}\right) \pm 2 \xi_{ \pm} / \omega} .
$$

Using the standard kinetic term for the $\mu$ field

$$
\mu=l^{-1} e^{-r / l}, \quad \partial \xi / \partial \mu=-l k_{-} \xi / \mu
$$

one gets the expression for the mass

$$
\begin{aligned}
m^{2} & =V_{\text {rad }}^{\prime \prime}\left(\xi_{ \pm}\right)\left(\frac{\partial \xi}{\partial \mu}\right)^{2} \\
& \approx 16 l^{-2} \lambda e^{\left(k_{-} l-2\right) r / l} \frac{\left(\xi_{ \pm}-1\right)\left(\xi_{-}-\xi_{+}\right)}{\left(\xi_{-}-\xi_{+}\right) \pm 2 \xi_{ \pm} / \omega}
\end{aligned}
$$


If $\xi_{-}$is not too close to unity the last factor becomes $\left(\xi_{ \pm}-1\right)$ in agreement with (69). On the other hand in the superpotential limit (39)

$$
\xi_{-} \rightarrow 1, \quad \xi_{+} \rightarrow 1-2 / \omega
$$

the last factor behaves as [using (89)]

$$
\frac{\left(\xi_{ \pm}-1\right)\left(\xi_{-}-\xi_{+}\right)}{\left(\xi_{-}-\xi_{+}\right) \pm 2 \xi_{ \pm} / \omega} \rightarrow \mp \frac{1}{\omega}
$$

in agreement with eq. (171). This shows that our fit is indeed consistent with the effective action (81).

The above parameterization is reasonable as long as $\xi_{+}<1$ which is the case we will consider hereafter. For the $\xi_{+}>1$ case on the one hand eqs. (51) and (52) imply $\Lambda_{+}<\Lambda_{\infty}$, so that $V_{\text {rad }}\left(\xi_{+}\right)$becomes negative and at the same time transforms into a minimum according to (69). In this case our Ansatz for the metric (2) does not allow for additional solutions between the two minima at $\xi_{ \pm}$while according to (69) any additional solution should be a minimum. Therefore our Ansatz in (2) produces in this case two local minima of the radion potential in configuration space but no local maximum. This complicates the question of what the radion potential might look like in the case $\xi_{+}>1$ so that we will disregard this case in the following.

\section{HOLOGRAPHIC PHASE TRANSITION}

In this section we will present the discussion of the holographic phase transition at finite temperature along the lines of refs. [10, 11, 13] ${ }^{12}$. At finite temperature the system allows for an additional gravitational solution with a black hole singularity in the bulk. This AdS-S metric describes in the AdS/CFT correspondence the high temperature phase of the system [24, 25]. This phase starts dominating at temperatures of the order of the TeV scale. In fact the potential difference between AdS-S and pure AdS phases is given by [10]

$$
-\left.4 \pi^{4}(M l)^{3} T_{h}^{4}\right|_{T_{h}=T}
$$

where $T_{h}$ is the scalar field parameterizing the distance between the horizon and UV brane and $T$ is the temperature of the system. On the other hand the difference between the

\footnotetext{
$\overline{12}$ A precursor at zero temperature can be found in $[23]$.
} 
RS-GW and pure AdS phases is expressed by eq. (159) which should equal eq. (95) at the critical temperature $T_{c}$ of the phase transition. It turns out that typically $T_{c}$ is between the electroweak and $\mathrm{TeV}$ scales ${ }^{13}$.

Starting from a hot universe in the AdS-S phase a first-order phase transition towards RS-GW may happen below $T_{c}$. In the five-dimensional picture this means that the IR brane emerges from the black hole horizon and to minimize the bounce action this crossing has to happen far from the UV brane. For this reason it is commonly assumed that the bounce path consists in moving the horizon away from the UV brane till arriving to the (unstable) pure AdS phase, and subsequently displacing the IR brane from $r=\infty$ to $r=r_{-}$. This reasonable assumption fixes the bounce path and it reduces the study of the tunneling probability to the usual analysis of the one-dimensional bounce [26, 27] in which the bouncing scalar field is identified with $\mu\left(T_{h}\right)$ in the part of the path between pure AdS and RS-GW (AdS-S) ${ }^{14}$.

In particular the path in the AdS-S space can be simplified by observing that the kinetic term of the field $T_{h}$ has a small pre-factor ${ }^{15}$. Consequently this part of the path becomes extremely short once $T_{h}$ is canonically normalized, so that in the AdS-S region the potential seen by the bouncing field can be approximated by a step function.

We will release the radion field $\mu$ from a certain initial position $\mu_{0}$ and we will evolve it to the point $\mu=0$ (corresponding to the pure AdS phase) according to the $O(3)$ bounce equation [26, 27]

$$
\partial_{\rho}^{2} \mu+2 \frac{\partial_{\rho} \mu}{\rho}=\partial_{\rho} V_{\text {rad }}
$$

where $\rho^{2}=\vec{x}^{2}$. In the bounce solution the radion field should arrive at $\mu=0$ with the kinetic energy necessary to jump and stop on the top of the AdS-S minimum

$$
4 \pi^{4} T^{4}=\left.6\left(\partial_{\rho} \mu\right)^{2}\right|_{\mu=0},
$$

and this solution is used to determine the bounce action $S_{3} / T$.

${ }^{13}$ In reporting the expression (95) we omit the subdominant contribution of the bulk matter fields since this correction plays a minimal role in our analysis. The same consideration holds for the thermal corrections to the RS-GW potential. We assume nearly all SM fields to be fundamental so that their thermal contribution to the two-phases free energies is similar and thus it cancels out.

14 For more details about the AdS-S solution and the bounce of this phase transition see refs. [10 13].

15 The estimate of this factor is controversial. We checked in the numerical evaluation that admitting a sizeable kinetic term would only lead to slightly smaller tunneling temperatures. 
In order to explicitly calculate the tunneling probability it is useful to rewrite the radion action (81) as

$$
S_{\text {eff }}=12(M l)^{3} \int d^{4} x \sqrt{\left|\operatorname{det} \bar{g}_{M N}\right|}\left(\frac{1}{2} \dot{\mu}^{2}-V_{\text {rad }}(\mu)\right),
$$

where the potential, neglecting a constant piece, is

$$
\begin{aligned}
V_{\text {rad }}(\mu)= & \hat{\lambda} \mu^{4} \frac{\omega(\omega+1)(\omega+2)}{\omega\left(1-\frac{\xi_{+}}{\xi_{-}}\right)-2 \frac{\xi_{+}}{\xi_{-}}}\left(\frac{\mu}{\mu_{-}}\right)^{-\omega l k_{-}-l k_{+}-l k_{-}} \\
& \times\left[\frac{1}{\omega+2}\left(\frac{\mu}{\mu_{-}}\right)^{-2 k_{-}}-\frac{1}{\omega+1}\left(\frac{\mu}{\mu_{-}}\right)^{-k_{-}}\left(1+\frac{\xi_{+}}{\xi_{-}}\right)+\frac{1}{\omega} \frac{\xi_{+}}{\xi_{-}}\right],
\end{aligned}
$$

with $\omega$ determined by eq. (89) and

$$
\begin{gathered}
\mu_{-}=l^{-1}\left(\frac{v_{2}}{v_{1}} \xi_{-}\right)^{-1 /\left(l k_{-}\right)}, \\
\hat{\lambda}=\frac{1}{24} \frac{v_{2}^{2}}{M^{3}}\left(l k_{-}\right)\left(l k_{+}\right)\left(\xi_{-}-1\right) \xi_{-} .
\end{gathered}
$$

The dimensionless tunneling action will only depend on the parameters $\hat{\lambda}, \omega$ and $\mu_{+} / \mu_{-}$ and not explicitly on $\mu_{-}$. This can be seen by using the conformal transformation

$$
x^{\mu} \rightarrow a^{-1} x^{\mu}, \quad V_{\text {rad }}(\mu) \rightarrow a^{-4} V_{\text {rad }}(a \mu)
$$

which is equivalent to a rescaling of all dimensionful quantities, in particular $\mu_{-} \rightarrow \mu_{-} / a$. Therefore the relevant scale involved in the bounce solution is $\mu_{0}$ and the functional determinant [26] of the tunneling process has to be proportional to $\mu_{0}^{4}$. Tunneling can hence occur for bubble action $S_{b}$

$$
S_{b} \simeq \log \frac{\mu_{0}^{4}}{\Lambda^{2}} \approx 4 \frac{r_{-}}{l}+4 \log \frac{\mu_{0}}{\mu_{-}} \lesssim 140
$$

where it is used that the expansion parameter is the cosmological constant of the RS-GW phase (52). Observe that if the tunneling takes place during radiation domination the Hubble expansion is quadratic in temperature and the right-hand side of this equation would increase when the tunneling temperature decreases. However in the present case the Hubble parameter is dominated by the vacuum energy (which can even lead to a short period of inflation [13, 27]) and a smaller temperature in fact lowers the right-hand side via its dependence in the functional determinant. Notice that even though the four-dimensional gravity 
contributions to the action are significant (due to the expansion) gravitational effects ${ }^{16}$ in the tunneling process should not relevantly modify our results [28].

As long as the release point is far away from the minimum and maximum of the potential

$$
\left(\xi_{+} / \xi_{-}\right)^{1 /\left|k_{-}\right|} \ll \frac{\mu_{0}}{\mu_{-}} \ll 1
$$

the field experiences a nearly conformal potential. If $\xi_{-}$is not too close to unity the position of the maximum, given by

$$
\left(\xi_{+} / \xi_{-}\right)^{-1 / l k_{-}} \lesssim\left(\frac{v_{2}}{v_{2}} \xi_{-}\right)^{-1 / l k_{-}}=e^{-r_{-} / l}=l \mu_{-}=\frac{\text { TeV scale }}{\text { Planck scale }}
$$

is such that there is a large hierarchy between the position of the minimum and the maximum and for a large range of release points the potential is nearly conformal.

In the conformal case the potential is of the form

$$
V_{\text {conf }} \rightarrow-\bar{\kappa} \mu^{4}
$$

Solving eqs. (96) and (97) with this potential gives an action, temperature and bubble size for the $O(3)$ symmetric bubble

$$
S_{3} / T \simeq 217.0 \bar{\kappa}^{-3 / 4}(M l)^{3}, \quad T / \mu_{0} \simeq 0.103 \bar{\kappa}^{1 / 4}, \quad \bar{\rho} \mu_{0} \simeq 3.45 \bar{\kappa}^{-1 / 2}
$$

However the potential (106) is normalized to the origin instead of being normalized at the AdS-S minimum [26] and we have to add to the action the omitted contribution

$$
\frac{16 \pi^{5}}{3} T^{3} \bar{\rho}^{3}(M l)^{3} \simeq 72.3 \bar{\kappa}^{-3 / 4}(M l)^{3}
$$

This yields for the tunnel action

$$
S_{3} / T \simeq 289.3 \bar{\kappa}^{-3 / 4}(M l)^{3}
$$

Besides thermal fluctuations the potential barrier can also be overcome by quantum fluctuations. In this case the bounce solution is $O(4)$ symmetric and eq. (96) has to be replaced by

$$
\partial_{\rho}^{2} \mu+3 \frac{\partial_{\rho} \mu}{\rho}=\partial_{\rho} V_{\text {rad }}
$$

16 Gravitational effects become important when nucleated bubbles are of order $1 / \sqrt{\Lambda}$. 
This tunnel configuration is relevant for temperatures that are below the inverse bubble radius [13, 27]. In the present model the bounce solution results to be in this regime and the quantum tunneling competes with thermal tunneling. In the conformal case, the solution to the bounce equation is given by

$$
\mu(\rho)=\frac{2 \mu_{0}}{2+\bar{\kappa} \rho^{2} \mu_{0}^{2}},
$$

which leads to the action

$$
S_{4}=12(M l)^{3} \frac{2 \pi^{2}}{3 \bar{\kappa}},
$$

while the corresponding temperature vanishes. Small deviations from the conformal case will only lead to a relatively small temperature while sizeable temperatures can only be obtained when the conformal symmetry is broken by a release point which is not too far away from the minimum of the potential. In this regime our nearly conformal approximation (112) underestimates the real action that could be reliably obtained in the thin-wall approximation. Still one can generally conclude that for the same release point the thermal tunneling leads to larger temperatures than the quantum tunneling since the friction term in the bounce equation is smaller. Hence if both tunneling modes are feasible the system tends to tunnel by thermal fluctuations.

In the near conformal case a good approximation is given by using a conformal potential normalized at the release point

$$
V_{\text {near-conf }}=V_{\text {rad }}\left(\mu_{0}\right)\left(\frac{\mu}{\mu_{0}}\right)^{4}
$$

such that

$$
\bar{\kappa}=-\frac{V_{r a d}\left(\mu_{0}\right)}{\mu_{0}^{4}} .
$$

In this approximation the minimum of the bounce action as a function of $\mu_{0}$ (or equivalently $T)$ is given by the maximum of $V_{\text {rad }}\left(\mu_{0}\right) / \mu_{0}^{4}$. According to (85) the function $V_{\text {rad }}(\mu) / \mu^{4}$ is nearly polynomial in terms of $\xi$ with one extremum between $\xi_{-}$and $\xi_{+}$. The derivative of this function at the endpoints is given by

$$
d_{ \pm}=\left.\partial_{\xi} \frac{V_{r a d}(\mu)}{\mu^{4}}\right|_{\xi=\xi_{ \pm}}=-\left.\frac{4}{l k_{-} \xi} \frac{V_{r a d}(\mu)}{\mu^{4}}\right|_{\xi=\xi_{ \pm}}=\frac{1}{6} \frac{v_{2}^{2}}{M^{3}}\left(l k_{+}\right)\left(\xi_{ \pm}-1\right) .
$$

A reasonable estimate of the position of the minimum is

$$
\frac{d_{-} \xi_{-}-d_{+} \xi_{+}}{d_{-}-d_{+}}=\xi_{-}+\xi_{+}-1,
$$


with the approximate value

$$
\bar{\kappa}_{\max }=-\frac{d_{-} d_{+}}{d_{-}-d_{+}} \frac{\xi_{-}-\xi_{+}}{2}=\frac{1}{12} \frac{v_{2}^{2}}{M^{3}}\left(l k_{+}\right)\left(\xi_{-}-1\right)\left(1-\xi_{+}\right)
$$

For example a nearly maximal value is given for $\xi_{-} \approx 1.5, v_{1} / v_{2} \approx 3$. In this case the coefficient is given by $\bar{\kappa} \approx 9.2 \times 10^{-3} v_{1}^{2} / M^{3}$. For this choice of parameters and with $v_{1}=$ $4 M^{3 / 2}, N=3, k_{-} l \simeq-0.019$, which create the correct hierarchy, a comparison between this approximation and the full numerical result is shown in fig. 2, It turns out that the estimate is quite reliable and in particular no appreciable difference is found for $S_{4}$.

The bulk cosmological constant is related to the five-dimensional Planck mass by the flux $N$ of the background fields which, according to the AdS/CFT correspondence, translates into the rank of the gauge group of the CFT in four dimensions. In the present context this relation is only known to leading order in $N$ and here we use $(M l)^{3}=\left(N^{2}-1\right) / 16 \pi^{2}$ as definition [29]. Then considering the maximal allowed $\bar{\kappa}$ for the thermal tunneling action one obtains the bound

$$
S_{3} / T \gtrsim 61.5 \times\left(N^{2}-1\right)\left(\frac{v_{1}^{2}}{M^{3}}\right)^{-3 / 4}
$$
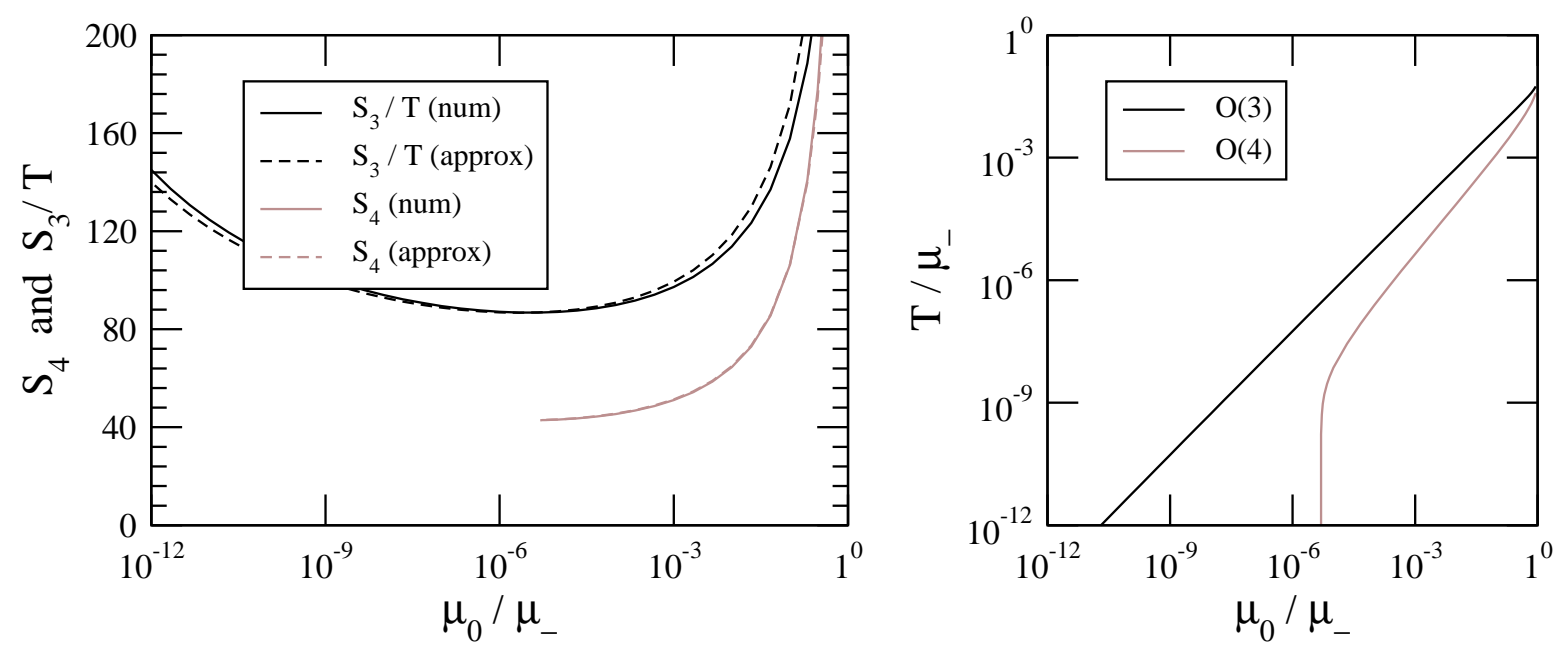

FIG. 2: The left plot shows the comparison of $S_{3} / T$ and $S_{4}$ as a function of the release point $\mu_{0}$ between the approximation in (113) and the full numerical results. The two curves for $S_{4}$ lie on top of each other and significant deviations only occur for a release point very close to the minimum of the potential. The right plot shows the temperature $T$ as a function of the release point $\mu_{0}$. The used values are $v_{1}=4 M^{3 / 2}, v_{2}=v_{1} / 3, N=3, \xi_{-}=1.5$ and $k_{-} l \simeq-0.019$. 
and a nucleation temperature that is substantially smaller than the scale $\mu_{-}$. Using the criterion (103) for tunneling this translates into

$$
N^{2}-1 \lesssim 2.3 \times\left(\frac{v_{1}^{2}}{M^{3}}\right)^{3 / 4} \quad \text { (thermal) }
$$

which is quite similar to the bound obtained in [10]. Analogously for the case of quantum tunneling one finds

$$
S_{4} \gtrsim 54.3 \times\left(N^{2}-1\right)\left(\frac{v_{1}^{2}}{M^{3}}\right)^{-1},
$$

and

$$
N^{2}-1 \lesssim 2.6 \times\left(\frac{v_{1}^{2}}{M^{3}}\right) \quad(\text { quantum })
$$

Depending on the value of $v_{1}^{2} / M^{3}$ the weakest bound between (119) and (121) constitutes the minimal requirement on $N$ allowing for a first-order transition.

Alternatively one can saturate the bound of applicability of our analysis (55) which reads, for $\xi_{-}$not too close to unity, as

$$
\frac{v_{2}^{2}}{M^{3}}\left(\xi_{-}-1-\frac{k_{-}}{k_{+}}\right)^{2} \lesssim \frac{3}{2 l^{2}},
$$

such that

$$
\bar{\kappa} \lesssim \frac{1}{2} \frac{\left(\xi_{-}-1\right)\left(1-\xi_{+}\right)}{\left(\xi_{-}-1-k_{-} / k_{+}\right)^{2}} \lesssim \frac{1}{2}
$$

which gives for thermal tunneling

$$
N \lesssim 6.8 \quad(\text { thermal })
$$

while for quantum tunneling one finds

$$
N \lesssim 12.5 \quad \text { (quantum) }
$$

Thus there is a small window where quantum tunneling is possible while thermal tunneling is not.

We conclude that even though the limits on tunneling derived including backreactions are parametrically similar to the results obtained without them our analysis shows that the constraint on the parameter space used in the literature, $\phi^{2} \ll M^{3}[6,10$ 13], is actually more conservative than necessary. 


\section{GRAVITATIONAL WAVE OBSERVATIONS}

The main input parameter for the determination of the gravitational wave spectrum is the inverse duration of the phase transition that, normalized to the Hubble parameter, is given by

$$
\beta / \sqrt{\Lambda}=T \frac{d}{d T}\left(\frac{S_{3}}{T}\right)
$$

This equation assumes that the temperature is proportional to the inverse scale factor, $T \propto \exp (-\sqrt{\Lambda} t)$, which is also true in the AdS-S phase [29]. In this section we will present analytic as well as numerical estimates for $\beta / \sqrt{\Lambda}$.

The dependence of the coefficient $\bar{\kappa}$ in the near-conformal case is approximately polynomial at the release point $\xi_{0}$. Besides the tunnel action should grow to infinity if $\xi_{0}$ approaches either $\xi_{-}$or $\xi_{+} \approx 2-\xi_{-}$. A reasonable fit is then given by

$$
\bar{\kappa}\left(\xi_{0}\right)=\bar{\kappa}_{\max }\left(1-\left(\frac{\xi_{0}-1}{\xi_{-}-1}\right)^{2}\right) .
$$

Hereby and depending on the model parameters the value of $\bar{\kappa}_{\max }$ is somewhere in the range

$$
\begin{array}{r}
3.1 \times 10^{-3}\left(N^{2}-1\right)^{4 / 3}<\bar{\kappa}_{\max }<0.56 \quad \text { (thermal) } \\
3.6 \times 10^{-3}\left(N^{2}-1\right)<\bar{\kappa}_{\max }<0.56 \quad \text { (quantum) }
\end{array}
$$

where the lower bound arises from the constraint that the system will tunnel [eqs. (109) and (112)] while the upper bound results from a numerical equivalent of (123) obtained by exploring the parameter space constrained by the requirement of small backreaction (55).

For the thermal tunneling action (109) the inverse duration of the phase transition is given by (notice that approximately $\mu_{0} \propto T$ as shown in fig. 2)

$$
\begin{aligned}
\beta / \sqrt{\Lambda} & =\frac{3}{4} \frac{S_{3}}{T}\left(l k_{-}\right) \frac{\xi_{0}}{\bar{\kappa}} \frac{d \bar{\kappa}}{d \xi_{0}}=\frac{3}{2} \frac{S_{3}}{T}\left(-l k_{-}\right) \frac{\bar{\kappa}_{\max }}{\bar{\kappa}} \frac{\xi_{0}}{\xi_{-}-1} \frac{\xi_{0}-1}{\xi_{-}-1} \\
& =\frac{3}{2} \frac{S_{3}}{T}\left(-l k_{-}\right) \frac{\xi_{0}}{\xi_{-}-1} \frac{\bar{\kappa}_{\max }}{\bar{\kappa}} \sqrt{1-\frac{\bar{\kappa}}{\bar{\kappa}_{\max }}} .
\end{aligned}
$$

At the first sight it seems that $\beta / \sqrt{\Lambda}$ can become arbitrarily large for $\xi_{-} \rightarrow 1$. However if $\xi_{-}$approaches the unity the constraint (54) becomes more severe than (55) and $\bar{\kappa}_{\max }$ is according to (117) bounded by

$$
\bar{\kappa}_{\text {max }} \simeq \frac{1}{12} \frac{v_{2}^{2}}{M^{3}}\left(l k_{+}\right)\left(\xi_{-}-1\right)\left(1-\xi_{+}\right)<\frac{2}{l k_{-}} \frac{v_{2}^{2}}{v_{1}^{2}}\left(\xi_{-}-1\right)\left(1-\xi_{+}\right),
$$


such that the maximum possible value of $\beta / \sqrt{\Lambda}$ in fact becomes constant in this limit. Hence one can obtain a conservative estimate by assuming that $\xi_{-}$is in the transition region where the two constraints (54) and (55) are equally severe

$$
\xi_{-}-1 \simeq \sqrt{-l k_{-}} \frac{1}{2} \frac{v_{1}}{v_{2}}
$$

and hence

$$
\beta / \sqrt{\Lambda}<3 \sqrt{-l k_{-}} \frac{S_{3}}{T} \frac{v_{2}}{v_{1}} \frac{\bar{\kappa}_{\max }}{\bar{\kappa}} \sqrt{1-\frac{\bar{\kappa}}{\bar{\kappa}_{\max }}}
$$

Noting that

$$
\sqrt{-l k_{-}} \frac{v_{2}}{v_{1}} \simeq \sqrt{-l k_{-}} e^{k_{-} r_{-}} \lesssim \sqrt{\frac{l}{2 e r_{-}}}
$$

one obtains the bound

$$
\beta / \sqrt{\Lambda} \lesssim \sqrt{\frac{9 l}{2 e r_{-}}} \frac{S_{3}}{T} \frac{\bar{\kappa}_{\max }}{\bar{\kappa}} \sqrt{1-\frac{\bar{\kappa}}{\bar{\kappa}_{\max }}} \approx 30 \frac{\bar{\kappa}_{\max }}{\bar{\kappa}} \sqrt{1-\frac{\bar{\kappa}}{\bar{\kappa}_{\max }}} .
$$

Eq. (128) for the thermal case implies

$$
\frac{\bar{\kappa}_{\max }}{\bar{\kappa}} \lesssim \frac{180}{\left(N^{2}-1\right)^{4 / 3}}
$$

which finally yields a bound on $\beta / \sqrt{\Lambda}$.

Analogously for quantum tunneling one finds

$$
\beta / \sqrt{\Lambda} \lesssim \sqrt{\frac{8 l}{e r_{-}}} S_{4} \frac{\bar{\kappa}_{\max }}{\bar{\kappa}} \sqrt{1-\frac{\bar{\kappa}}{\bar{\kappa}_{\max }}}\left(\frac{T}{\mu_{0}} \frac{d \mu_{0}}{d T}\right) .
$$

The last factor is typically smaller than in thermal tunneling (where it is unity, cf. fig. 2) so that commonly quantum tunneling happens only when the system cannot tunnel by thermal fluctuations, which implies

$$
\bar{\kappa}_{\text {max }}<\left(\frac{140}{290} 16 \pi^{2}\right)^{-4 / 3}\left(N^{2}-1\right)^{4 / 3} .
$$

Besides the largest $\bar{\kappa}$ that can be realized in the present model is generally given by $\bar{\kappa}_{\max }<$ 0.56 according to eq. (128). On the other hand the quantum tunneling condition (128) implies

$$
\bar{\kappa}>(2 \times 140)^{-1}\left(N^{2}-1\right),
$$

such that successful quantum transitions which are not spoiled by earlier thermal tunneling lead to the constraint

$$
\frac{\bar{\kappa}_{\max }}{\bar{\kappa}} \lesssim \min \left[0.85\left(N^{2}-1\right)^{1 / 3}, 155\left(N^{2}-1\right)^{-1}\right] .
$$


The first bound increases with $N$ and arises from the requirement of no thermal tunneling while the second bound stems from viable quantum tunneling and decreases with $N$. As a result, as far as $N$ decreases the quantum tunneling bounds on $\beta / \sqrt{\Lambda}$ become first weaker (while thermal tunneling becomes less likely) and then stronger (as thermal tunneling is in general not possible for $N>7$ ). Finally for $N>12$ tunneling is impossible altogether.

The approximate values and numerical results on the bound on $\beta / \sqrt{\Lambda}$ are given in Table【. Already for $N=2$ one observes a rather strong phase transition $(\alpha \gtrsim 1)$. We see that for small $N$, when the release point is close to the minimum of the potential, our approximation overestimates the numerical result for $\beta / \sqrt{\Lambda}$, while for larger values of $N$ it yields fairly precise results.

The second ingredient for the gravity wave spectrum is the vacuum energy normalized to the radiation energy of the system (traditionally denoted by $\alpha$ in the literature)

$$
\alpha=\frac{12(M l)^{3}\left(V_{\text {rad }}(\infty)-V_{\text {rad }}\left(r_{-}\right)\right)}{\rho_{\text {radiation }}}=\frac{l^{-2} \Lambda}{2 \pi^{4} T_{n}^{4}} .
$$

In the present system this parameter is much larger than unity as it is shown in Table \due to the large supercooling. This also implies that the nucleated bubbles expand with near

\begin{tabular}{|c|c|c|c|c|c|}
\hline & \multicolumn{3}{|c|}{ thermal } & \multicolumn{2}{|c|}{ quantum } \\
\hline$N$ & $\beta_{\text {approx }} / \sqrt{\Lambda}$ & $\beta_{\text {num }} / \sqrt{\Lambda}$ & $\alpha_{\text {num }}$ & $\beta_{\text {num }} / \sqrt{\Lambda}$ & $\alpha_{\text {num }}$ \\
\hline 2 & $<1230$ & $<770$ & $>3.2$ & $<15$ & $>1.0 \times 10^{11}$ \\
\hline 3 & $<235$ & $<315$ & $>10$ & $<33$ & $>5 \times 10^{7}$ \\
\hline 4 & $<131$ & $<143$ & $>50$ & $<45$ & $>2.5 \times 10^{6}$ \\
\hline 5 & $<62$ & $<67$ & $>800$ & $<56$ & $>4 \times 10^{5}$ \\
\hline 6 & $<29$ & $<30$ & $>10^{5}$ & $<63$ & $>1.3 \times 10^{5}$ \\
\hline 7 & $<6.5$ & $<6.0$ & $>10^{8}$ & $<71$ & $>5 \times 10^{4}$ \\
\hline 8 & & & & $<54$ & $>5 \times 10^{5}$ \\
\hline 9 & & & & $<40$ & $>8 \times 10^{6}$ \\
\hline 10 & & & & $<27$ & $>3 \times 10^{8}$ \\
\hline 11 & & & & $<17$ & $>4 \times 10^{10}$ \\
\hline 12 & & & & $<5.4$ & $>5 \times 10^{14}$ \\
\hline
\end{tabular}

TABLE I: Upper limits on $\beta / \sqrt{\Lambda}$ and lower limits on $\alpha$ for all possible values of $N$. 
luminal velocities. The vacuum energy is very efficiently transformed into bulk motion of the plasma or directly into kinetic energy of the bubble wall and resides in a very thin shell around the bubble wall [30]. In this sense the phase transition is extremely strong.

The main mechanisms of gravity wave production during a first-order phase transition are bubble collisions [31-36], turbulence [37-40] and magneto-hydrodynamic turbulence [41, 42]. In our analysis we will focus on the production mechanism by bubble collisions for the following reasons. First, while reliable information about the gravity wave spectrum in the case of bubble collisions is provided by computer simulations, the analysis of turbulence relies typically on additional assumptions (e.g. on the overall normalization of the spectrum). Second, the peak frequency of the spectrum produced by turbulence compared to the one by bubble collisions is typically suppressed by the eddy velocity that occurs in the turbulent fluid motion [37, 38]. At the same time the spectrum for turbulence falls off faster than the one by bubble collisions. Hence even if the different components are of similar size they might be disentangled because they lead to a double peak structure or at least a knee in the spectrum. Finally, recent studies have shown that very strong phase transitions most probably have a runway behavior of the wall [43] and in this case most of the vacuum energy is transformed into gradient/kinetic energy of the Higgs and not into collective bulk motion of the plasma [30]. This should also reduce the portion of energy that leads to turbulent plasma motion. In the following we will focus on the spectrum produced by bubble collisions.

In the above discussed limit, $\alpha \gg 1$, the energy fraction for colliding bubbles of the gravitational radiation at the time of production is given by [36]

$$
h^{2} \Omega^{*}=7.7 \times 10^{-2}\left(\frac{\sqrt{\Lambda}}{\beta}\right)^{2} .
$$

In a standard cosmology this energy density is diluted to today's observed energy fraction

$$
h^{2} \bar{\Omega}=1.3 \times 10^{-6}\left(\frac{\sqrt{\Lambda}}{\beta}\right)^{2} .
$$

The only assumptions that enters here is that the Universe is dominated by radiation after the phase transition. Immediately after the phase transition the energy fraction stays constant up to the time of matter-radiation equality when the energy fraction of gravitational radiation starts to become suppressed together with all the other light components of the plasma. 
The frequency peak of the spectrum at time of generation is in the limit of very strong phase transition, $\alpha \gg 1$, and large wall velocities, $v_{b} \sim 1$, given by [36]

$$
f^{*}=0.23 \beta .
$$

The red-shift to today's observed spectrum depends on the reheating temperature after the phase transition according to

$$
\bar{f}=0.23 \beta \frac{T_{0}}{T_{r e h}},
$$

where $T_{0}$ is the observed temperature of the cosmic microwave background. The spectrum is of form [36]

$$
\Omega(f)=\bar{\Omega} \frac{3.8 f^{2.8} \bar{f}^{1.0}}{1.0 \bar{f}^{3.8}+2.8 f^{3.8}} .
$$

Several sample spectra are given in Fig. 3.

As long as the expansion is negligible during the phase transition the reheating temperature $T_{r e h}$ can be determined by energy conservation. Since the phase transition is extremely supercooled at the typical nucleation temperatures the energy density of AdS-S phase is comparable to the one of pure AdS and thus

$$
g_{*} \frac{\pi^{2}}{30} T_{\text {reh }}^{4} \simeq 12(M l)^{3}\left(V_{\text {rad }}(0)-V_{\text {rad }}\left(\mu_{-}\right)\right)=6(M l)^{3} l^{-2} \Lambda,
$$

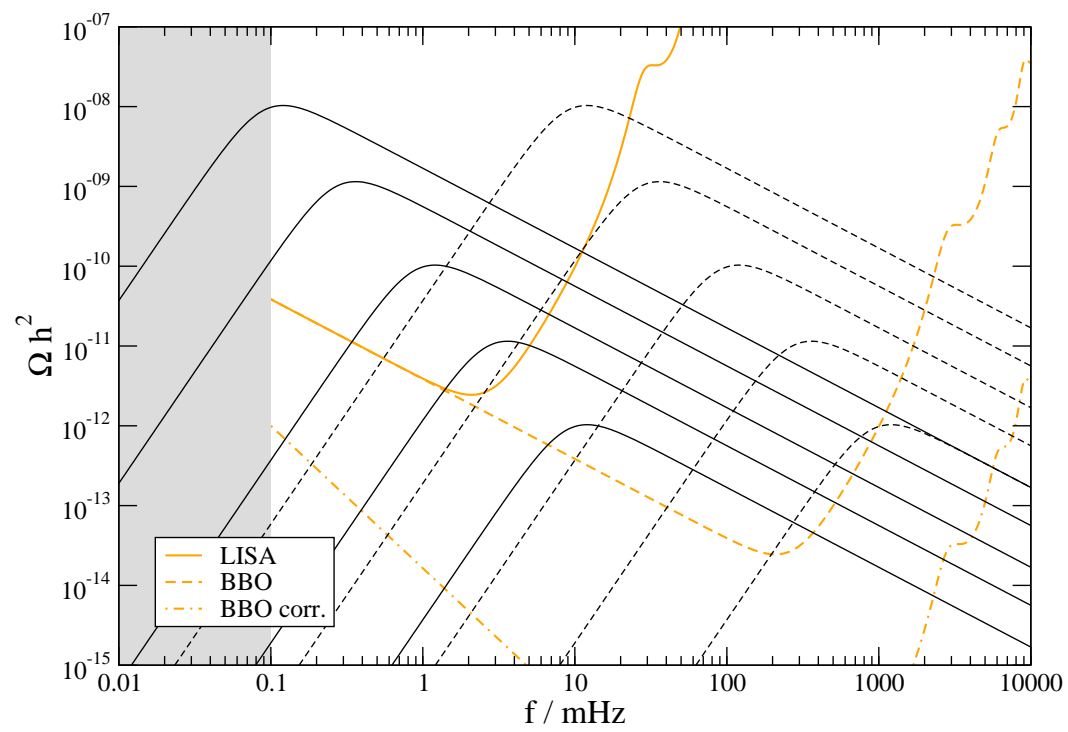

FIG. 3: Several example spectra of gravitational waves. The straight (dashed) lines are for a reheating temperature $T_{r e h} / \sqrt{\Lambda}=10^{16}\left(T_{r e h} / \sqrt{\Lambda}=10^{14}\right)$. From bottom to top the plots use $\beta / \sqrt{\Lambda}=\{1000,300,100,30,10\}$. The sensitivities of the LISA and BBO experiments are taken from [44]. 
where $\Lambda=\Lambda_{\infty}$ and $g_{*}$ denotes the effective number of degrees of freedom just after the phase transition. Notice that for $g_{*} \lesssim 120 \pi^{2}(M l)^{3}$ the critical temperature, given by

$$
4 \pi^{4} T_{c}^{4}=6 l^{-2} \Lambda,
$$

is smaller than the reheating temperature and percolation is followed by a period of phase coexistence ${ }^{17}$. Nevertheless we will assume that the red-shift of the gravity waves is given by the naive expression involving the reheating temperature (146).

We now give a conservative estimate for the range of possible values for $T_{r e h} / \sqrt{\Lambda}$ in a realistic model. According to (52) and (69) the expansion parameter is related to the radion mass by

$$
\Lambda \approx \frac{1}{8} m_{\text {rad }}^{2} e^{-2 r_{-} / l},
$$

such that together with the definition of the four-dimensional Planck mass, $\left(M_{P} l\right)^{2}=(M l)^{3}$, one obtains

$$
g_{*} \frac{\pi^{2}}{30} \frac{T_{r e h}^{4}}{\Lambda^{2}}=48 \frac{M_{P}^{2}}{m_{\text {rad }}^{2}} e^{2 r / l} .
$$

Introducing the $\mathrm{TeV}$ scale $\mu_{-}=l^{-1} e^{-r_{-} / l}$ and the relation $\left(M_{P} l\right)^{2}=\left(N^{2}-1\right) / 16 \pi^{2}$ the peak frequency can be written as

$$
\bar{f}=1.77 \times 10^{-3} \mathrm{mHz} \frac{\beta}{\sqrt{\Lambda}}\left(N^{2}-1\right)^{1 / 4} \sqrt{\frac{m_{\text {rad }} \mu_{-}}{\mathrm{TeV}^{2}}} .
$$

Realistically the last factor varies within a range of $[0.3,10]$. The corresponding ranges for the possible positions of the peak structure of the gravitational wave spectrum compared with sensitivities of future experiments are shown in Fig. 4. For $N \leq 3$ the phase transition could be weak enough to prohibit detection by LISA. Besides for these values the $1 / N$ expansion is clearly questionable and stringy loop contributions invalidate our analysis. On the other hand for $N \geq 4$ the model would lead to a gravitational wave signal that is observable by LISA.

\section{CONCLUSION}

Motivated by the sizeable impact of backreactions on the radion mass determined with the so-called superpotential method [15, 16], we studied backreactions to the radion potential in a perturbative scheme. Our approach includes backreactions on the five-dimensional

${ }^{17}$ Assuming that some SM fields be composite relaxes the condition on $g_{*}$. 


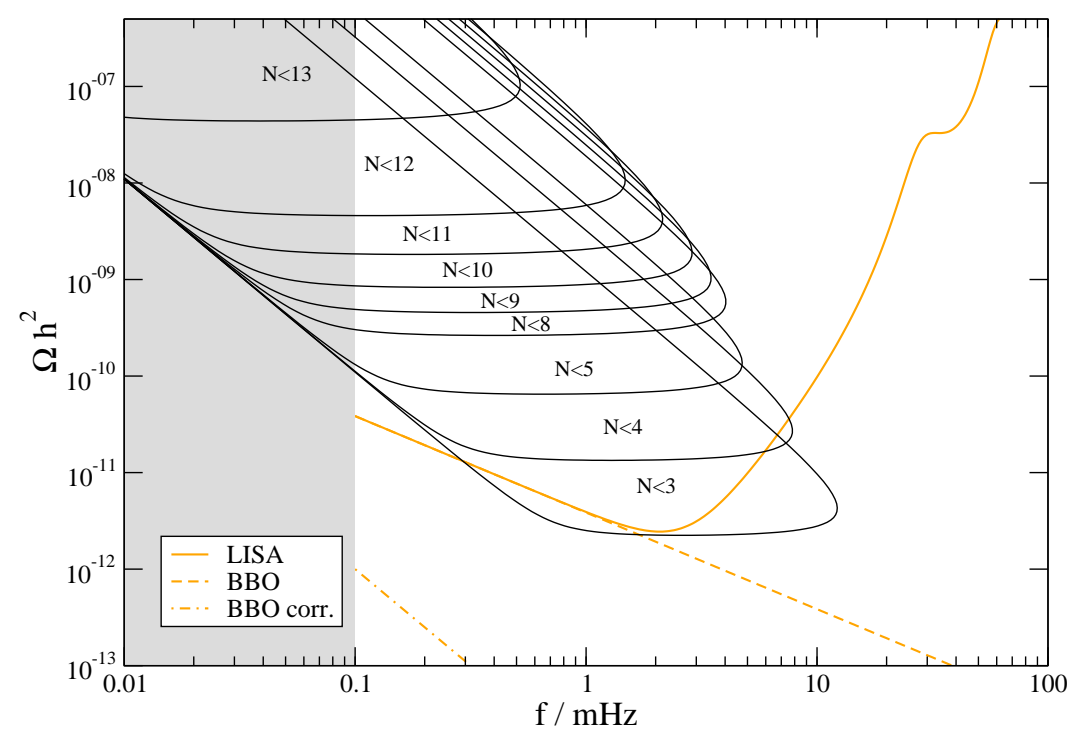

FIG. 4: The regions denote the possible positions for the peak of the gravity wave spectrum depending on the parameter $N$. The signal will be detected by LISA, BBO or BBO correlated when it stands above their respective lines.

gravitational fields from the bulk scalar under the constraints in eqs. (54) and (55) which are less severe than the usual assumption of weak fields, $\phi^{2} \ll M^{3}$ [6, 10 13]. An immediate consequence of the backreactions is the necessity to generalize the metric to include the Hubble expansion of space-time in four dimensions. In our framework a judicious choice of brane tensions allows to reproduce the radion potential found by Goldberger and Wise as well as (within the applicability of our approach) the radion mass obtained with the superpotential method. We then clarify the apparent paradox that the radion mass computed in the GW and superpotential frameworks scale (for a bulk scalar mass $m^{2} \sim 4 k_{-} / l$ ) as $\left|k_{-}\right|^{3 / 2}$ and $\left|k_{-}\right|^{2}$, respectively. On top of that in the regime of sizeable detuning of brane tensions we found that a scaling proportional to $k_{-}$is possible. This results in a larger radion mass for fixed $\mathrm{TeV}$ scale and in turn it leads to a deeper radion potential which has significant impact on the thermal phase transition of the system.

The phase transition constitutes a serious problem for the holographic interpretation of models with a radion stabilized by a bulk scalar [10 14]: the tunnel action scales as $N^{2}$ with the flux $N$ of the background field such that the metastable symmetric phase becomes, for large values of $N$, effectively stable and the conformal symmetry is never broken during the course of the Universe. One possible solution to this problem is to consider more general 
scalar potentials (as e.g. done in ref. [45]) which modify the behavior of the bulk fields considerably in the IR. Analogously large backreactions can have potentially the same effect by decreasing the 'effective' $N$ through a stronger warping close to the TeV brane.

In our analysis of the phase transition, and compared to former work [10, 11], we did not use the common thick or thin wall approximations to obtain the tunnel action but devised an approximation that is tailor-made for nearly-conformal potentials. Besides notice that we used the kinetic term obtained by decoupling the Einstein equations [15] which differs by a factor of 2 from the one used in [10, 11, 13]. The main consequence of our approach is that the tunneling action depends logarithmically on the temperature and can lead to a couple of e-folds of low scale inflation without tuning the model parameters (this was already observed in the numerical analysis of ref. [13]). While a few e-folds of inflation cannot solve the horizon problem a low phase transition temperature has a large impact on gravitational wave production since, in this case, the energy stored in the vacuum bubbles during percolation at the end of the phase transition is many orders of magnitude larger than the energy stored in the thermal plasma.

In summary we found that, in the regime of large backreactions, the deeper radion potential leads to a significantly weaker phase transition and numerically the absolute limit $N<13$ applies. Besides we reanalyzed the gravitational wave spectrum produced by the first-order phase transition. We conclude that as long as stringy corrections can be neglected (specifically $N>3$ ), the model leads to a stochastic background of gravitational radiation that can be observed by LISA (see fig. (4)).

\section{Acknowledgments}

Work supported in part by the European Commission under the European Union through the Marie Curie Research and Training Network "UniverseNet" (MRTN-CT-2006-035863); by the Spanish Consolider-Ingenio 2010 Programme CPAN (CSD2007-00042); by CICYT, Spain, under contract FPA 2008-01430; by the Belgian IISN convention 4.4514.08 of FNRS; and by ERC starting grant Cosmo@LHC. 


\section{Appendix A: Linearized system of equations and the radion mass}

In order to calculate the radion mass we will follow the analysis of ref. [15] including the expansion of the Universe. For the perturbations of the metric we use the Ansatz

$$
\begin{aligned}
\phi(x, r) & =\phi_{0}(r)+\varphi(x, r), \\
d s^{2} & =e^{2 A+2 F(x, r)} \bar{g}_{\mu \nu} d x^{\mu} d x^{\nu}-(1-2 F(x, r))^{2} d r^{2},
\end{aligned}
$$

where $\bar{g}$ denotes the induced $d S_{4}$ metric as in eq. (2)

$$
\bar{g}_{\mu \nu} d x^{\mu} d x^{\nu}=d t^{2}-e^{2 \sqrt{\Lambda} t}\left(d x_{1}^{2}+d x_{2}^{2}+d x_{3}^{2}\right)
$$

and solve the linearized Einstein equations, $\delta R_{\mu \nu}=\delta T_{\mu \nu}$. The corresponding entries for the Riemann tensor are given by

$$
\begin{aligned}
& \delta R_{\mu \nu}=\bar{g}_{\mu \nu}\left(-\square F+F^{\prime \prime}+10 A^{\prime} F^{\prime}+6 A^{\prime \prime} F+24 A^{\prime 2} F\right), \\
& \delta R_{\mu 5}=-6 A^{\prime} \partial_{\mu} F-3 \partial_{\mu} F^{\prime}, \\
& \delta R_{55}=-2 \square F-16 A^{\prime} F^{\prime}-4 F^{\prime \prime},
\end{aligned}
$$

where we defined the d'Alembertian operator in curved space time $\left(\bar{g}=\operatorname{det} \bar{g}_{\mu \nu}\right)$

$$
\square F=\bar{g}^{-\frac{1}{2}} \partial_{\mu}\left(\bar{g}^{\frac{1}{2}} \bar{g}^{\mu \nu} \partial_{\nu} F\right)=\bar{g}^{\mu \nu} \partial_{\mu} \partial_{\nu} F+3 \sqrt{\Lambda} e^{-2 A} \partial_{t} F
$$

The energy-momentum tensor is given by

$$
\begin{aligned}
\delta T_{\mu \nu}= & -\frac{4}{3} \bar{g}_{\mu \nu}\left(V^{\prime}\left(\phi_{0}\right) \varphi+2 V\left(\phi_{0}\right) F\right) \\
& -\frac{2}{3} \bar{g}_{\mu \nu} \sum_{i}\left(\frac{\partial \lambda_{i}\left(\phi_{0}\right)}{\partial \phi} \varphi+4 \lambda_{i}\left(\phi_{0}\right) F\right) \delta\left(r-r_{i}\right), \\
\delta T_{\mu 5}= & 2 \phi_{0}^{\prime} \partial_{\mu} \varphi \\
\delta T_{55}= & 4 \phi_{0}^{\prime} \varphi^{\prime}+\frac{4}{3} V^{\prime}\left(\phi_{0}\right) \varphi-\frac{16}{3} V\left(\phi_{0}\right) F \\
& +\frac{8}{3} \sum_{i}\left(\frac{\partial \lambda_{i}\left(\phi_{0}\right)}{\partial \phi} \varphi-2 \lambda_{i}\left(\phi_{0}\right) F\right) \delta\left(r-r_{i}\right),
\end{aligned}
$$

and the scalar equation is given by

$$
\begin{aligned}
& \square \varphi-\varphi^{\prime \prime}-4 A^{\prime} \varphi^{\prime}+\frac{\partial^{2} V}{d \phi^{2}}\left(\phi_{0}\right) \varphi= \\
& \quad-\sum_{i}\left(\frac{\partial \lambda_{i}\left(\phi_{0}\right)}{\partial \phi} \varphi-2 \lambda_{i}\left(\phi_{0}\right) F\right) \delta\left(r-r_{i}\right)-6 \phi_{0}^{\prime} F^{\prime}-4 \frac{\partial V}{\partial \phi} F .
\end{aligned}
$$


The equation for $R_{\mu 5}$ can be integrated to yield

$$
\phi_{0}^{\prime} \varphi=-\frac{3}{2}\left(F^{\prime}+2 A^{\prime} F\right) .
$$

Next consider the equation $\frac{1}{4} \bar{g}^{\mu \nu} \delta R_{\mu \nu}+\delta R_{55}$ in the bulk

$$
3 \square F+6 A^{\prime} F^{\prime}-24 A^{2} F-6 A^{\prime \prime} F+3 F^{\prime \prime}=8 V F-4 \phi_{0}^{\prime} \varphi^{\prime} .
$$

With eqs. (A7)

$$
-\frac{2}{3} \phi_{0}^{\prime} \varphi^{\prime}=F^{\prime \prime}+2 A^{\prime \prime} F+2 A^{\prime} F^{\prime}-\frac{\phi_{0}^{\prime \prime}}{\phi_{0}^{\prime}}\left(F^{\prime}+2 A^{\prime} F\right)
$$

and eqs. (9)-(10)

$$
-\frac{4}{3} V=A^{\prime \prime}+4 A^{2}-3 \Lambda e^{-2 A}
$$

this can be brought into the form

$$
\begin{aligned}
\square F= & F^{\prime \prime}+2 A^{\prime} F^{\prime}+4 A^{\prime \prime} F-2 \frac{\phi_{0}^{\prime \prime}}{\phi_{0}^{\prime}}\left[F^{\prime}+2 A^{\prime} F\right] \\
& +6 \Lambda e^{-2 A} F .
\end{aligned}
$$

A minimally coupled scalar in four-dimensional curved space time fulfills the equation

$$
\left(e^{2 A} \square+m^{2}\right) F=0,
$$

which leads to the equation

$$
\left(-m^{2}-6 \Lambda\right) e^{-2 A} F=F^{\prime \prime}+2 A^{\prime} F^{\prime}+4 A^{\prime \prime} F-2 \frac{\phi_{0}^{\prime \prime}}{\phi_{0}^{\prime}}\left[F^{\prime}+2 A^{\prime} F\right] .
$$

Hence in an expanding Universe the sole difference on the equation for the radion mass with respect to the usual radion equation can be taken into account by a shift in the mass parameter

$$
\hat{m}^{2}=m^{2}+6 \Lambda \text {. }
$$

Since the Hubble parameter is many orders smaller than the radion mass this shift can quite generally be neglected. Using the Ansatz $F=e^{2 A} \hat{F}$ this leads to

$$
\hat{F}^{\prime \prime}+2 A^{\prime \prime} \hat{F}-2 A^{\prime} \hat{F}^{\prime}-2 \frac{\phi_{0}^{\prime \prime}}{\phi_{0}^{\prime}} \hat{F}^{\prime}=-\hat{m}^{2} e^{-2 A} \hat{F},
$$

which is the starting point of the analysis in section IVB.

[1] L. Randall and R. Sundrum, "A large mass hierarchy from a small extra dimension," Phys. Rev. Lett. 83 (1999) 3370 arXiv:hep-ph/9905221; ;An alternative to compactification," Phys. Rev. Lett. 83 (1999) 4690 arXiv:hep-th/9906064. 
[2] J. M. Cline, C. Grojean, G. Servant, "Cosmological expansion in the presence of extra dimensions," Phys. Rev. Lett. 83 (1999) 4245 hep-ph/9906523.

[3] C. Csaki, M. Graesser, L. Randall and J. Terning, "Cosmology of brane models with radion stabilization," Phys. Rev. D 62 (2000) 045015 arXiv:hep-ph/9911406].

[4] J. Garriga, O. Pujolas, T. Tanaka, "Radion effective potential in the brane world," Nucl. Phys. B605 (2001) 192-214 hep-th/0004109].

[5] J. Garriga, A. Pomarol, "A Stable hierarchy from Casimir forces and the holographic interpretation," Phys. Lett. B560 (2003) 91-97 hep-th/0212227.

[6] W. D. Goldberger and M. B. Wise, "Modulus stabilization with bulk fields," Phys. Rev. Lett. 83 (1999) 4922 arXiv:hep-ph/9907447].

[7] J. M. Maldacena, "The large N limit of superconformal field theories and supergravity," Adv. Theor. Math. Phys. 2 (1998) 231 [Int. J. Theor. Phys. 38 (1999) 1113] [arXiv:hep-th/9711200]; S. S. Gubser, I. R. Klebanov and A. M. Polyakov, "Gauge theory correlators from non-critical string theory," Phys. Lett. B 428 (1998) 105 arXiv:hep-th/9802109; E. Witten, "Anti-de Sitter space and holography," Adv. Theor. Math. Phys. 2 (1998) 253 arXiv:hep-th/9802150.

[8] R. Contino, Y. Nomura, A. Pomarol, "Higgs as a holographic pseudoGoldstone boson," Nucl. Phys. B671 (2003) 148-174 hep-ph/0306259.

[9] N. Arkani-Hamed, A. G. Cohen, H. Georgi, "Electroweak symmetry breaking from dimensional deconstruction," Phys. Lett. B513 (2001) 232-240 hep-ph/0105239.

[10] P. Creminelli, A. Nicolis and R. Rattazzi, "Holography and the electroweak phase transition," JHEP 0203 (2002) 051 arXiv:hep-th/0107141.

[11] L. Randall and G. Servant, "Gravitational Waves from Warped Spacetime," JHEP 0705 (2007) 054 arXiv:hep-ph/0607158.

[12] J. Kaplan, P. C. Schuster and N. Toro, "Avoiding an empty universe in RS I models and large-N gauge theories," arXiv:hep-ph/0609012.

[13] G. Nardini, M. Quiros and A. Wulzer, "A Confining Strong First-Order Electroweak Phase Transition," JHEP 0709 (2007) 077 [arXiv:0706.3388 [hep-ph]].

[14] M. Reece and L. T. Wang, "Randall-Sundrum and Strings," arXiv:1003.5669 [hep-ph].

[15] C. Csaki, M. L. Graesser and G. D. Kribs, "Radion dynamics and electroweak physics," Phys. Rev. D 63 (2001) 065002 arXiv:hep-th/0008151.

[16] O. DeWolfe, D. Z. Freedman, S. S. Gubser and A. Karch, "Modeling the fifth dimen- 
sion with scalars and gravity," Phys. Rev. D 62 (2000) 046008 arXiv:hep-th/9909134. PHRVA,D62,046008;

[17] J. W. York, "Role of conformal three geometry in the dynamics of gravitation," Phys. Rev. Lett. 28 (1972) 1082.

[18] G. W. Gibbons and S. W. Hawking, "Action Integrals And Partition Functions In Quantum Gravity," Phys. Rev. D 15 (1977) 2752.

[19] N. Arkani-Hamed, S. Dimopoulos, N. Kaloper and R. Sundrum, "A small cosmological constant from a large extra dimension," Phys. Lett. B 480 (2000) 193 arXiv:hep-th/0001197.

S. Kachru, M. B. Schulz and E. Silverstein, "Self-tuning flat domain walls in 5d gravity and string theory," Phys. Rev. D 62 (2000) 045021 arXiv:hep-th/0001206.

S. Forste, Z. Lalak, S. Lavignac and H. P. Nilles, "A comment on self-tuning and vanishing cosmological constant in the brane world," Phys. Lett. B 481 (2000) 360 arXiv:hep-th/0002164. C. Csaki, J. Erlich, C. Grojean and T. J. Hollowood, "General properties of the self-tuning domain wall approach to the cosmological constant problem," Nucl. Phys. B 584 (2000) 359 arXiv:hep-th/0004133.

S. H. H. Tye and I. Wasserman, "A brane world solution to the cosmological constant problem," Phys. Rev. Lett. 86 (2001) 1682 arXiv:hep-th/0006068.

J. M. Cline and H. Firouzjahi, "A small cosmological constant from warped compactification with branes," Phys. Lett. B 514 (2001) 205 arXiv:hep-ph/0012090.

[20] W. D. Goldberger and M. B. Wise, "Phenomenology of a stabilized modulus," Phys. Lett. B 475 (2000) 275 arXiv:hep-ph/9911457.

[21] R. Rattazzi and A. Zaffaroni, "Comments on the holographic picture of the Randall-Sundrum model," JHEP 0104 (2001) 021 arXiv:hep-th/0012248.

[22] Robert M. Wald, General Relativity, The University of Chicago Press, Chicago 60637, 1984.

[23] J. M. Cline and H. Firouzjahi, "Brane-world cosmology of modulus stabilization with a bulk scalar field," Phys. Rev. D 64 (2001) 023505 [arXiv:hep-ph/0005235].

[24] S. W. Hawking and D. N. Page, "Thermodynamics Of Black Holes In Anti-De Sitter Space," Commun. Math. Phys. 87 (1983) 577.

[25] E. Witten, "Anti-de Sitter space, thermal phase transition, and confinement in gauge theories," Adv. Theor. Math. Phys. 2 (1998) 505 arXiv:hep-th/9803131].

[26] S. R. Coleman, "The Fate Of The False Vacuum. 1. Semiclassical Theory," Phys. Rev. D 15 
(1977) 2929 [Erratum-ibid. D 16 (1977) 1248]; C. G. Callan and S. R. Coleman, "The Fate Of The False Vacuum. 2. First Quantum Corrections," Phys. Rev. D 16 (1977) 1762.

[27] A. D. Linde, "Fate of the False Vacuum at Finite Temperature: Theory and Applications," Phys. Lett. B100 (1981) 37.

[28] S. R. Coleman and F. De Luccia, "Gravitational Effects On And Of Vacuum Decay," Phys. Rev. D 21 (1980) 3305.

[29] S. S. Gubser, "AdS/CFT and gravity," Phys. Rev. D 63 (2001) 084017 arXiv:hep-th/9912001].

[30] J. R. Espinosa, T. Konstandin, J. M. No and G. Servant, "Energy Budget of Cosmological First-order Phase Transitions," arXiv:1004.4187 [hep-ph].

[31] A. Kosowsky, M. S. Turner and R. Watkins, "Gravitational Radiation From Colliding Vacuum Bubbles," Phys. Rev. D 45 (1992) 4514.

[32] A. Kosowsky, M. S. Turner and R. Watkins, "Gravitational waves from first order cosmological phase transitions," Phys. Rev. Lett. 69 (1992) 2026.

[33] A. Kosowsky and M. S. Turner, "Gravitational Radiation From Colliding Vacuum Bubbles: Envelope Approximation To Many Bubble Collisions," Phys. Rev. D 47 (1993) 4372 arXiv:astro-ph/9211004].

[34] M. Kamionkowski, A. Kosowsky and M. S. Turner, "Gravitational radiation from first order phase transitions," Phys. Rev. D 49 (1994) 2837 arXiv:astro-ph/9310044.

[35] C. Caprini, R. Durrer and G. Servant, "Gravitational wave generation from bubble collisions in first-order phase transitions: an analytic approach," Phys. Rev. D 77 (2008) 124015 arXiv:0711.2593 [astro-ph]].

[36] S. J. Huber and T. Konstandin, "Gravitational Wave Production by Collisions: More Bubbles," JCAP 0809 (2008) 022 arXiv:0806.1828 [hep-ph]].

[37] A. Kosowsky, A. Mack and T. Kahniashvili, "Gravitational radiation from cosmological turbulence," Phys. Rev. D 66 (2002) 024030 arXiv:astro-ph/0111483.

[38] A. D. Dolgov, D. Grasso and A. Nicolis, "Relic backgrounds of gravitational waves from cosmic turbulence," Phys. Rev. D 66 (2002) 103505 arXiv:astro-ph/0206461.

[39] G. Gogoberidze, T. Kahniashvili and A. Kosowsky, "The spectrum of gravitational radiation from primordial turbulence," Phys. Rev. D 76 (2007) 083002 [arXiv:0705.1733 [astro-ph]].

[40] C. Caprini, R. Durrer, T. Konstandin, G. Servant, "General Properties of the Gravitational Wave Spectrum from Phase Transitions," Phys. Rev. D 79 (2009) 083519 arXiv:0901.1661 
[astro-ph]].

[41] C. Caprini and R. Durrer, "Gravitational waves from stochastic relativistic sources: Primordial turbulence and magnetic fields," Phys. Rev. D 74 (2006) 063521 arXiv:astro-ph/0603476.

[42] C. Caprini, R. Durrer and G. Servant, "The stochastic gravitational wave background from turbulence and magnetic fields generated by a first-order phase transition," JCAP 0912 (2009) 024 arXiv:0909.0622 [astro-ph.CO]].

[43] D. Bodeker, G. D. Moore, "Can electroweak bubble walls run away?," JCAP 0905 (2009) 009. arXiv:0903.4099 [hep-ph]].

[44] A. Buonanno, G. Sigl, G. G. Raffelt, H. T. Janka and E. Muller, "Stochastic gravitational wave background from cosmological supernovae," Phys. Rev. D 72 (2005) 084001 arXiv:astro-ph/0412277]; and http://www.srl.caltech.edu/ shane/sensitivity.

[45] B. Hassanain, J. March-Russell and M. Schvellinger, "Warped Deformed Throats have Faster (Electroweak) Phase Transitions," JHEP 0710 (2007) 089 [arXiv:0708.2060 [hep-th]]. 